Revue européenne des migrations internationales

vol. $19-n^{\circ} 3 \mid 2003$

Moyen-Orient : mutations récentes d'un carrefour migratoire

\title{
Migrations internationales et politique en Jordanie
}

Françoise de Bel-Air

\section{(2) OpenEdition}

Journals

Édition électronique

URL : https://journals.openedition.org/remi/2651

DOI : 10.4000/remi.2651

ISSN : $1777-5418$

Éditeur

Université de Poitiers

Édition imprimée

Date de publication : 12 décembre 2003

Pagination : 9-41

ISBN : 2-911627-35-0

ISSN : 0765-0752

Référence électronique

Françoise de Bel-Air, "Migrations internationales et politique en Jordanie », Revue européenne des

migrations internationales [En ligne], vol. $19-n^{\circ} 3 \mid 2003$, mis en ligne le 20 juin 2006, consulté le 14 avril 2022. URL : http://journals.openedition.org/remi/2651 ; DOI : https://doi.org/10.4000/remi.2651

Ce document a été généré automatiquement le 14 avril 2022.

(c) Université de Poitiers 


\title{
Migrations internationales et politique en Jordanie
}

\author{
Françoise de Bel-Air
}

1 En Jordanie, le recours à l'émigration internationale a constitué, et constitue encore de nos jours, une stratégie de stabilisation politique et sociale ayant garanti la reproduction des structures du pouvoir. Cette stratégie a répondu aux mouvements de migrations forcées de 1948 et 1967 en provenance de Palestine, mais aussi à celui de 1990-1991, né de la Guerre du Golfe. Ces trois mouvements ont en effet chacun profondément affecté le contexte économique, social et politique jordanien, en particulier le mode de redistribution des ressources et d'accès au capital.

2 L'instrumentalisation des mouvements migratoires dans la pratique politique après la Guerre du Golfe ne peut être démontrée sans un retour sur le contexte préliminaire à cette période. Les caractéristiques de la population jordanienne et des relations Étatsociété, les contraintes rencontrées dans l'accès au capital, qui perdurent en Jordanie jusqu'à nos jours, trouvent en effet leur source dans les exodes palestiniens de 1948 et 1967. Les modalités du recours à l'émigration internationale, de 1973 au milieu des années 1980, pour répondre à ces contraintes particulières ont, de même, été modelées par le contexte né de ces mouvements. Dans un premier temps, pour la période 1973-1985, nous examinerons donc le rôle de l'émigration comme réponse à l'impossibilité, pour les réfugiés et déplacés de Palestine, d'accéder au capital en Jordanie. Nous l'envisagerons aussi comme stratégie de contrôle politique et économique de la part de l'État, à travers le développement de l'État rentier. A partir de 1985 , les flux migratoires de retour vers la Jordanie s'intensifiant, nous développerons les conséquences de ce phénomène sur les relations État-société et sur les modalités de l'accès au capital des citoyens. Dans un second temps, nous montrerons que les nouvelles revendications touchant à la participation politique et à la redistribution des ressources en ayant découlé sont, encore une fois, évacuées par le recours aux migrations internationales à partir de 1994. Nous nous concentrerons dans cet article sur les aspects directement politiques du contexte et de l'impact des migrations. Nous n'y traiterons pas de leur rôle dans le processus de changement social ${ }^{1}$. 
Deux décennies de migrations massives : essor et chute de l'état rentier en Jordanie

L'afflux de 300000 personnes vers la Jordanie, à la suite de la Guerre du Golfe de 1990-1991 augmente de près de $10 \%$ la population jordanienne. Cet événement entraîne une pression très forte sur les infrastructures de services et sur les logements. Il déséquilibre le maillage urbain sur le territoire. Au plan politique, il déstabilise le processus de redistribution des ressources et du capital, déjà profondément affecté par une crise économique ayant frappé le pays à la fin des années 1980. Les retours massifs des returnees ${ }^{2}$ du Golfe ont donc précipité la fin du cycle de développement d'un État rentier et d'un État providence, qui avaient assuré la stabilité politique du régime hachémite. Le développement de l'État rentier avait été rendu possible par la hausse des prix du pétrole en 1973, qui avait stimulé une demande massive de main-d'œuvre des États du Golfe producteurs d'hydrocarbures. Cette opportunité migratoire peut donc être vue comme le facteur ayant permis la survie du régime hachémite en Jordanie, menacée par l'afflux des réfugiés de Palestine en 1948 et 1967.

Migrations forcées : contraintes de développement, tensions politiques et sociales

Les mouvements massifs d'émigration vers les pays du Golfe en 1973 ont en effet été stimulés par un contexte particulièrement tendu, aux plans économique, politique et social.

5 La création de l'État d'Israël en 1948 et la guerre israélo-arabe en résultant auraient conduit plus de 700000 Palestiniens à l'exode, dont 70000 à 100000 ont trouvé refuge sur la rive est du Jourdain, habitée à cette époque par 480000 personnes environ. La population de la Jordanie aurait donc subi une augmentation de $20 \%$. Ayant annexé officiellement la Cisjordanie en avril 1950, le royaume «comportait à présent une population totale de près d'un million et demi d'âmes, dont moins d'un demi-million de Transjordaniens, tout le reste étant palestinien " (Salibi, 1996 : 246).

6 A la suite de la guerre des Six-Jours en juin 1967, la Jordanie perd la Cisjordanie, passée ainsi que la bande de Gaza sous occupation militaire israélienne. 395000 personnes rejoignent la rive est pendant et après le conflit (Samha, 1990: 215): des réfugiés, déplacés une seconde fois de la Cisjordanie à la Jordanie, des réfugiés de la bande de $\mathrm{Gaza}^{4}$, mais aussi des non-refugiés, anciens résidents de la bande de Gaza et de Cisjordanie, les « déplacés " ${ }^{5}$.

7 Ce triplement de la population jordanienne entre 1952 et 1979 (tableau 1) fait peser de lourdes contraintes de développement sur la Jordanie. Le développement urbain est intense $^{6}$ et incontrôlé : il entraîne une "sur-urbanisation » au profit de la région nord. Cette distribution inégale des infrastructures, des investissements et des services sous l'effet de l'afflux des réfugiés et déplacés accentue la rupture entre ville et campagnes, entre le sud et le nord du pays.

Tableau 1 : Population de la Jordanie, 1952-1994

\begin{tabular}{|c|c|c|c|}
\hline & rive est & rive ouest & total \\
\hline 1952 & 586200 & 742828 & 1329028 \\
\hline 1961 & 900800 & 805450 & 1706250 \\
\hline 1979 & 2133000 & - & 2133000 \\
\hline 1994 & 4139000 & - & 4139000 \\
\hline
\end{tabular}

Source : Recensements de population 
D'autre part, la précarité des conditions de vie des réfugiés et déplacés ${ }^{7}$, l'écart croissant entre riches et pauvres sont amplifiées par la nature non productive de l'économie: la Jordanie est privée de ressources naturelles et d'industries, ce qui la rend massivement dépendante des aides étrangères pour sa subsistance. Le triplement de sa population en un quart de siècle rend difficile le partage de ces maigres ressources ${ }^{8}$.

Mais surtout, les difficultés d'accès au capital résultent de la structure clientéliste du système de redistribution des ressources établi depuis la création de l'Émirat ${ }^{9}$. Le chômage va en s'accroissant depuis la perte de la Cisjordanie. Les tentatives de pénétration ou d'ascension au sein du marché du travail local se heurtent à la mainmise des élites installées avant 1948 et à celle des bureaucrates enrichis par la croissance rapide de l'économie avant 1967. Cette situation rend difficile l'accès des réfugiés et déplacés à la prospérité en Jordanie (Czichowski, 1990 : 149). Leur capital social, acquis dans un autre contexte et difficilement transposable ${ }^{10}$, ne leur permet donc pas d'accéder aux ressources. Cette situation alimente leur frustration, en particulier celle des diplômés ${ }^{11}$.

Les revendications en faveur d'une redistribution plus équitable et les tensions politiques se fondent non seulement sur le verrouillage du système de redistribution, mais aussi sur la question de l'effectif numérique de la composante palestinienne en Jordanie. Outre une pression sur les infrastructures, le triplement des effectifs en vingtcinq ans sous l'effet des migrations forcées engendre une vive inquiétude au sein de la population installée en Jordanie avant 1948. Ce facteur soulève en effet la question de l'allocation du pouvoir en fonction de la part respective des deux composantes (ou perçues comme telles) de la population, alors que l'identité palestinienne acquiert peu à peu une dimension politique ${ }^{12}$. Les effectifs de réfugiés et déplacés au sein de la population jordanienne ne sont pas publiés ${ }^{13}$, afin de ne pas porter atteinte à la cohésion nationale. On sait pourtant qu'ils ont dû atteindre environ $50 \%$ de la population totale à partir de $1967^{14}$.

11 Pour l'ensemble de ces raisons, cette massive présence de Jordano-Palestiniens inquiète la base de soutien transjordanienne du régime. L'instabilité politique croissante régnant en Jordanie à partir de cette date, la crise économique et la hausse du chômage provoquent un état de crise continue de 1967 à $1971^{15}$.

L'émigration comme régulateur politico-économique : une politique de l'État jordanien?

12 Dans ce contexte, l'ouverture des marchés du Golfe à une immigration de travail massive à partir de 1973 représente un débouché à l'impasse économico-politique dans laquelle se trouve la Jordanie. En outre, l'afflux des remises des travailleurs expatriés vers le Golfe va entraîner une rentiérisation de l'État (ou de l'économie), un système générateur d'importantes ressources, pour la population mais aussi pour l'État.

13 Entre 1950 et 1973, des flux migratoires au départ de la Jordanie des deux rives compensaient déjà quelque peu l'absence d'incorporation des migrants forcés dans la société jordanienne. Les exodes de 1948 et de 1967 se prolongeaient en migrations de travail et se dirigeaient, d'une part, vers les pays occidentaux, d'autre part, vers les pays arabes producteurs de pétrole. 
Après la guerre de 1973 et la hausse des prix du pétrole ayant suivi, l'ampleur de la crise économique et politique en Jordanie est à la mesure de celle des flux de migrants quittant le pays pour les marchés du Golfe (figure 1$)^{16}$.

Figure 1 : Les migrations en Jordanie

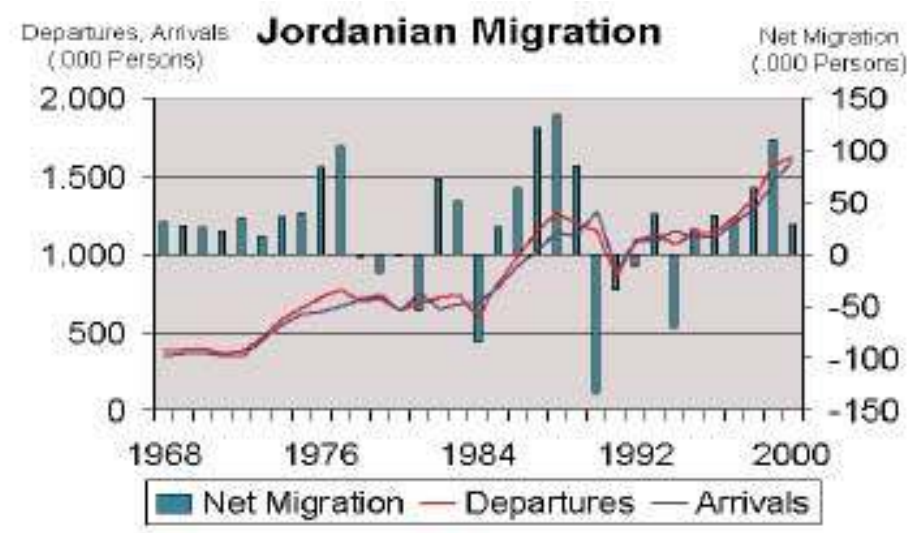

Ce graphique est emprunté à ZAQQA, 2001

(1) expulsé du pays. L'alinéa suivant indique que «aucun Jordanien ne peut être empêché de résider dans aucun lieu, ou forcé d'y résider, sauf dans des circonstances prescrites par la loi ${ }^{17}$. La liberté de migrer hors des frontières n'est pas mentionnée explicitement, mais G. Dib, dans son analyse des lois régissant les migrations interrégionales au Moyen-Orient (Dib, 1979a ; b) remarque que, tous les pays de la région étant membres des NationsUnies, ils se sentiraient ainsi moralement forcés d'accorder ce droit à leurs citoyens. 
19 La politique éducative joue également un rôle propre à pousser la main-d'œuvre jordanienne sur les marchés du Golfe. Les pays importateurs de main-d'œuvre appliquant des règles régissant l'entrée, la sortie, les conditions d'emploi des immigrés ${ }^{18}$, les pays exportateurs, tels la Jordanie, ont rapidement tenté de s'adapter aux besoins des employeurs potentiels. Ces politiques sont élaborées en relation avec le marché du travail dans le Golfe, où l'émigration de travail, au moins officiellement, était considérée comme temporaire ${ }^{19}$, et sans doute plus propice au retour des remises vers la Jordanie. Le niveau moyen d'éducation élevé des émigrés des deux rives facilite leur insertion sur le marché du travail du Golfe. La formation d'une main-d'œuvre adaptée aux besoins des pays exportateurs de pétrole reste, comme en témoignent les Plans de développement de 1970 à 1990, le seul élément publiquement discuté et affirmé d'une politique de gestion des migrations ; les efforts se concentrent d'ailleurs particulièrement sur le développement de l'enseignement technique.

Enfin, si la Jordanie n'a pas développé d'institution propre à encadrer ces courants sur le long terme, un réseau administratif minimal est mis en place. Depuis 1976, les ambassades jordaniennes à l'étranger disposent d'un personnel spécialisé dans les questions d'emploi; depuis 1981, le ministère du Travail jordanien a ouvert une « section des émigrants » (Share', 1991; Russel, 1988).

21 Pour pallier les menaces parfois émises par les pays récepteurs de main-d'œuvre, qui stigmatisent volontiers le recours à une force de travail immigrée comme un phénomène de nature temporaire et soumis à leur bon vouloir (Luciani et Salamé, 1988), quelques mesures sont adoptées par la Jordanie. Le Plan de Développement quinquennal de 1981-85 appelle en effet à «engager des accords bilatéraux avec les pays arabes et autres, afin de réguler les flux de sortie de la main-d'œuvre jordanienne » (HKJ / NPC, 1981: 298). D'autre part, remettant en cause l'idée d'une émigration entièrement laissée à l'initiative individuelle et familiale des migrants, divers ministères (Éducation) et institutions (Armée) ouvrent la voie à l'émigration pour des membres du secteur public, par le biais des détachements à l'étranger par contrat avec leurs homologues des pays d'accueil (Émirats Arabes Unis, Oman, Arabie Saoudite, ...) ${ }^{20}$ (Seccombe, 1987).

Cette "politique de la porte ouverte" et les mesures d'encadrement des flux migratoires mises en place portent leurs fruits. A partir des années soixante-dix, l'expatriation de certains de leurs membres permet aux familles un meilleur accès au capital en Jordanie. Également, les montants élevés de l'aide au développement versée par les pays accueillant la main-d'œuvre jordanienne permettent une reproduction des structures du pouvoir. L'émigration garantit donc au régime jordanien une sécurité, politique autant qu'économique.

23 En effet, à l'issue de ces mouvements, $40 \%$ de la population active employée jordanienne travaille hors des frontières au milieu des années quatre-vingt (Samha, 1999 : 3). Le taux de chômage est ramené de plus de 12 \% durant les années cinquante et soixante (Samha, 1990) à $2 \%$. Un début de pénurie de main-d'œuvre se fait même jour à partir du milieu des années soixante-dix, dans les secteurs de la construction et de l'agriculture. L'accès au capital s'en trouve facilité pour les personnes restées en Jordanie.

$24 \mathrm{Au}$ plan politique, la part des réfugiés et des déplacés dans la population totale diminue, car les migrations touchent principalement les zones urbaines et les populations d'origine palestinienne. Il est impossible de désagréger les données concernant ces dernières de celles touchant aux personnes installées en Jordanie avant 
$1948^{21}$, et seules les données du recensement de 1961 fournissent des indications sur la répartition des migrants selon l'origine géographique et la destination. Les personnes originaires de Palestine représentaient alors $80 \%$ du total des migrants jordaniens. Dans les pays arabes, elles constituaient $82 \%$ du total des migrants (HKJ, 1964). Au Koweit seul, $95 \%$ des Jordaniens étaient d'origine palestinienne au cours des années quatre-vingt (Gorokhoff, 1985 ; Brand, 1988 ${ }^{22}$ ).

Concernant les émigrés eux-mêmes, expatriés en raison de leurs difficultés d'accès au capital en Jordanie (capital économique, capital social, voire capital politique), leurs signes d'enracinement dans les pays du Golfe, par exemple à Koweit, traduisent le succès de cette stratégie (Gorokhoff, 1985 ; Longuenesse, 1991; Fargues, 1991). En 1990-1991, parmi les migrants revenus du Golfe au cours de l'année, 70 \% avaient passé plus de trente ans à l'étranger (NCERD, 1991).

Mais plus encore, l'émigration des travailleurs jordaniens vers les pays du Golfe est directement liée à l'attribution des aides arabes à la Jordanie, versées par les pays exportateurs de pétrole et importateurs de main-d'œuvre. Ces revenus contribuent à faire de la Jordanie un État rentier et lui donnent les moyens de contrôler le processus de redistribution des ressources et du capital.

La politique de laissez-faire à l'égard de l'émigration permet d'éviter des tractations avec les riches pays arabes, qui, afin de garantir la stabilité du pays et la régularité des flux de main-d'œuvre se sont engagés, par exemple lors de la Conférence de Bagdad en 1978, à verser à la Jordanie plus d'un milliard de dollars par an pendant dix ans (Owen, 1983). Le tableau 2 indique que, même si ces sommes n'ont en fait jamais été versées intégralement, l'aide arabe à la Jordanie représente, jusqu'au milieu des années quatrevingt, une part irremplaçable de son PIB. Ce système économique, contrôlé, selon la plupart des auteurs, par l'État, entraîne l'application du qualificatif d'État rentier à la Jordanie ${ }^{23}$. 
Tableau 2 : Montant annuel des aides versées à la Jordanie et leur part dans le PIB (1961-1983)

\begin{tabular}{|c|c|c|c|c|c|c|c|}
\hline & $\begin{array}{c}\text { Aide } \\
\text { arabe }\end{array}$ & $\begin{array}{c}\text { Aide } \\
\text { USA }\end{array}$ & $\begin{array}{c}\text { Autres aides } \\
\text { publiques }\end{array}$ & $\begin{array}{c}\text { Aides } \\
\text { privées }\end{array}$ & $\begin{array}{c}\text { Total aides } \\
\text { étrangères }\end{array}$ & $\begin{array}{c}\text { \% aides } \\
\text { étrangères } \\
\text { dans PIB }\end{array}$ & $\begin{array}{c}\text { Aides } \\
\text { per } \\
\text { capita }\end{array}$ \\
\hline & (millions J.D.) & (millions J.D. & (millions J.D.) & (millions J.D.) & (millions J.D.) & (\%) & (J.D.) \\
\hline 1961 & - & 17,1 & 8,3 & 0,9 & 26,2 & 20,6 & 15,4 \\
\hline 1962 & - & 15,5 & 8,0 & 1,7 & 25,2 & 19,3 & 14,4 \\
\hline 1963 & - & 15,5 & 7,0 & 1,6 & 24,1 & 17,5 & 13,3 \\
\hline 1964 & 4,5 & 15,0 & 7,0 & 2,0 & 28,5 & 17,8 & 15,3 \\
\hline 1965 & 7,3 & 12,0 & 7,5 & 2,7 & 29,5 & 16,3 & 15,4 \\
\hline 1966 & 9,5 & 13,4 & 8,6 & 2,7 & 34,1 & 18,4 & 17,3 \\
\hline 1967 & 37,6 & 7,6 & 6,4 & 2,4 & 53,9 & 26,2 & 26,4 \\
\hline 1968 & 46,3 & 1,2 & 5,6 & 1,4 & 54,5 & 27,6 & 25,9 \\
\hline 1969 & 41,2 & 1,3 & 3,4 & 1,6 & 47,4 & 20,3 & 21,6 \\
\hline 1970 & 33,1 & 1,4 & 4,6 & 1,6 & 40,7 & 18,3 & 17,7 \\
\hline 1971 & 19,1 & 12,8 & 3,6 & 1,1 & 36,6 & 15,5 & 15,4 \\
\hline 1972 & 23,2 & 36,0 & 6,8 & 2,3 & 68,3 & 26,0 & 27,8 \\
\hline 1973 & 23,7 & 30,8 & 6,6 & 3,5 & 64,6 & 22,2 & 25,4 \\
\hline 1974 & 46,6 & 25,3 & 12,5 & 2,3 & 86,7 & 23,2 & 33,1 \\
\hline 1975 & 105,7 & 22,4 & 9,9 & 1,8 & 139,8 & 37,2 & 51,8 \\
\hline 1976 & 77,6 & 26,1 & 19,0 & 3,8 & 126,6 & 22,5 & 45,5 \\
\hline 1977 & 132,3 & 15,6 & 19,0 & $-1,1$ & 165,8 & 25,1 & 57,4 \\
\hline 1978 & 66,3 & 18,6 & 17,8 & 3,9 & 106,5 & 13,6 & 35,7 \\
\hline 1979 & 299,7 & 4,6 & 13,8 & $-3,6$ & 314,4 & 34,1 & 101,8 \\
\hline 1980 & 370,4 & 6,2 & 14,3 & 7,9 & 398,8 & 33,6 & 123,1 \\
\hline 1981 & 394,9 & - & 20,4 & 15,5 & 430,8 & 28,7 & 128,2 \\
\hline 1982 & 335,8 & - & 27,9 & 9,6 & 373,3 & 22,0 & 107,6 \\
\hline 1983 & 258,3 & & 31,8 & 5,4 & 295,4 & 16,0 & 82,4 \\
\hline
\end{tabular}

Source : Central Bank of Jordan

28 Garantie du revenu rentier de l'État et de sa répartition, l'émigration génère également un revenu par le biais des remises des travailleurs émigrés. $\mathrm{Si}$, depuis la création de l'Émirat, l'État restait dépendant d'une rente ${ }^{24}$, à partir de 1973-1975 la situation évolue par l'afflux des biens privés constitués par les remises. L. Brand parle de l'émergence d'une économie rentière (Brand, 1995). Grâce à l'émigration des années 1970, ce sont donc l'État et l'économie qui se « rentiérisent ».

Le recours soutenu aux aides étrangères et la rentiérisation de l'État permettent au régime de contrôler les modes de formation des groupes d'intérêt et de pression, constitués en fonction du type de revenus dont ils dépendent (Anderer, 1991). Au plan politique, la rente alimente les réseaux clientélistes et stabilise ainsi la base de soutien du régime. Pour la population, la part importante des remises des travailleurs émigrés dans le PIB (Tableau 3) compense les effets du clientélisme et la nature improductive de l'économie. La hausse des revenus des familles entraînée par l'afflux des remises a permis une progression du niveau de vie et la création d'une société de consommation en Jordanie, par l'accroissement de la masse monétaire disponible (Czichowski, 1990 ; 1991 ; RSS, 1986, Share', 1987, etc.). Les remises sont pour une part essentielle utilisées à l'achat de biens de consommation (70 à $80 \%$ selon Findlay et Samha, 1986), à l'éducation des enfants, au logement, et non à des investissements productifs, mais ceci évite en retour une rationnalisation de l'économie, déstabilisante pour le système de redistribution existant ${ }^{25}$.

30 L'émigration de Jordanie vers les pays producteurs de pétrole du Golfe, entre 1973 et 1985, est donc à envisager comme un facteur de stabilisation politico-économique. Les facteurs de tension, liés aux revendications d'accès au pouvoir émises par les JordanoPalestiniens sur la base de leur poids numérique et au verrouillage de l'accès au capital économique, politique et social de cet État "patrimonial», ont été contournés par 
l'élargissement du marché du travail et surtout par la possibilité de débouchés pour les diplômés. L'émigration vers le Golfe a offert une opportunité de mobilité sociale aux personnes écartées des groupes d'intérêt et du processus de la redistribution des richesses en Jordanie. La rentiérisation de l'État et de l'économie découlant de ces mouvements constituent donc la clé de voûte de la reproduction des structures du pouvoir, aux plans politique et social.

Renversement des flux migratoires et faillite du contrat social

31 Mais l'émergence de migrations de retour du Golfe vers la Jordanie, à partir de 1985, auxquelles ont succédé une série d'événements politiques et économiques, va profondément affecter ce relatif équilibre. Devant la remise en cause des modalités de la redistribution du capital, des revendications seront exprimées, touchant à une plus grande participation des acteurs sociaux au processus de décision et de redistribution des ressources. Pourtant, paradoxalement, ces revendications n'émaneront pas des migrants de retour, «volontaires » dès 1985 ou forcés hors des pays du Gulf Cooperation Council $^{26}$ et de l'Irak par la crise du Golfe, mais des régions rurales du pays, jusqu'alors peu touchées par les migrations internationales. La passivité politique des migrants d'autre part, l'absence d'institutions intermédiaires, détentrices et redistributrices de capital, capables d'influencer le processus de développement, en partenariat avec l'État ou hors de sa sphère d'influence, suggèrent en outre l'échec du contrat social entre l'État et les citoyens, de même que la difficulté croissante pour l'ensemble de la population d'accéder au capital.

Migrations de retour, nouvel afflux de réfugiés : l'émergence d'une crise économique et politique en Jordanie

32 Durant la première moitié des années quatre-vingt, la chute des prix sur le marché du pétrole et la baisse des revenus pétroliers entraînent une récession au sein des économies des pays producteurs et, en conséquence, une diminution de l'aide arabe versée à la Jordanie. Plus important, les politiques mises en place dans les pays du Golfe ont entraîné le remplacement progressif de la main-d'œuvre arabe par une maind'œuvre asiatique. L'explication invoquée par les chercheurs reste de nature politique et sécuritaire, les premiers renvoyés étant les Chi'ites du Sud-Liban et les Palestiniens (Feiler, 1993).

En conséquence, même si les flux migratoires nets en Jordanie restent négatifs jusqu'au déclenchement de la Guerre du Golfe en 1990, le nombre de travailleurs émigrants jordaniens cesse d'augmenter de façon marquée dès 1983-1984 : 339000 en 1987 (dont 277000 dans les pays arabes), une baisse de 4000 à 5000 travailleurs migrants par an par rapport à la phase précédente (World Bank, 1994). Outre ce ralentissement, des flux de retour font leur apparition. Au Koweit seul, le nombre de Jordaniens et de Palestiniens avait décru de 50000 entre 1985 et la mi-1990 (Russel, 1992).

Une enquête démographique menée en 1986 sur les migrants de retour vers la Jordanie (HKJ, 1986) montre que la plus grande part d'entre eux (44\%) étaient revenus d'Arabie Saoudite. Seuls $15 \%$ rentraient du Koweit, où l'on a vu que l'implantation des migrants jordaniens était plus ancienne, durable et familiale. Les premiers rentrés étaient donc des migrants plus tardifs, moins installés à l'étranger, plus à-même de disposer d'un capital en Jordanie qu'à l'étranger. Ces migrants restaient en effet dépendants de leurs employeurs dans leur lieu d'immigration: la majorité d'entre eux étaient revenus principalement en raison de l'expiration de leurs contrats d'emploi (30\% des cas), mais aussi, pour $15 \%$ d'entre eux, à cause de mauvaises conditions de travail. 
35 À la suite de ces migrations de retour, le début de la décennie quatre-vingt-dix voit le troisième exode (ici en majorité) palestinien vers le Royaume en moins de quarante ans. De 200 à 300000 returnees rentrent précipitamment entre 1990 et 1992.

La crise du Golfe de 1990-1991 avait donné lieu à un déplacement de population de très grande ampleur à l'échelle de la région. On estime à quatre, voire à cinq millions l'effectif total des personnes déplacées, originaires de pays arabes (Palestiniens et Jordaniens, mais aussi Yéménites et Soudanais), de l'Asie du sud et du sud-est, tous employés jusqu'alors dans les pays du Golfe et en Irak. Environ un million de ces déplacés ont transité par la Jordanie, avant que les ressortissants de pays tiers ne soient rapatriés en quelques semaines vers leur pays d'origine (Van Hear, 1994b). Finalement, la Jordanie a conservé sur son sol 300000 de ces déplacés, des ressortissants jordaniens qui, pour la plupart, n'avaient jamais vécu dans ce pays.

37 Cette situation a eu de très fortes répercussions sur le pays d'accueil. La population jordanienne était estimée en 1990 à 3,5 millions de personnes (Banque mondiale, 1992 ; HKJ, Annuaire statistique, 1995). Sur la base d'un effectif de 300000 personnes ayant quitté le Golfe pour la Jordanie, l'afflux des returnees augmente donc de 8 à $9 \%$ l'effectif total de la population nationale. Le choc démographique, malgré une certaine réémigration vers la rive ouest et l'étranger est donc durement ressenti, d'autant que les returnees se concentrent à $95 \%$ dans la région nord du pays (Amman, Zarqa, Irbid). Ils intensifient ainsi le déséquilibre de la répartition spatiale de la population.

Mais surtout, l'impact démographique des returnees se manifeste sur la structure communautaire de la population, en reportant à près des $50 \%$ la part des JordanoPalestiniens dans la population totale du Royaume. On a vu en effet qu'une part estimée à $95 \%$ des Jordaniens du Koweit, la sous-population la plus nombreuse au sein des returnees, était d'origine palestinienne (Brand, 1988).

$39 \mathrm{Au}$ plan politique, cette situation annule l'effet des migrations sur la structure communautaire de la population (Jordaniens installés avant 1948 / réfugiés, déplacés de Palestine et leurs descendants). Elle éveille donc les protestations des nationalistes jordaniens et ravive les tensions politiques, dans un contexte de domination fragilisée par la faillite de l'État rentier, donc des structures de redistribution.

40 Les flux de remise provenant des travailleurs expatriés ont en effet diminué. Après un pic en 1984, la contribution au PIB de ces fonds décroît régulièrement, jusqu'à tomber sous la barre des $10 \%$ en $1991^{27}$ (Tableau 3). 
Tableau 3 : Montant des remises des émigrés et leur part dans le PIB (1961-1998)

\begin{tabular}{|c|c|c|c|c|c|}
\hline année & $\begin{array}{c}\text { Remises } \\
\text { des émigrés } \\
\text { (millions J.D.) }\end{array}$ & $\begin{array}{c}\text { \% remises } \\
\text { dans le } \\
\text { PNB } \\
\text { (\%) }\end{array}$ & année & $\begin{array}{c}\text { Remises } \\
\text { des émigrés } \\
\text { (millions J.D.) }\end{array}$ & $\begin{array}{c}\text { \% remises } \\
\text { dans le } \\
\text { PNB } \\
\text { (\%) }\end{array}$ \\
\hline $\mathbf{1 9 6 1}$ & 5,3 & 4,2 & $\mathbf{1 9 8 0}$ & 236,7 & 19,9 \\
\hline $\mathbf{1 9 6 2}$ & 6,2 & 4,7 & $\mathbf{1 9 8 1}$ & 340,9 & 23,0 \\
\hline $\mathbf{1 9 6 3}$ & 6,2 & 4,5 & $\mathbf{1 9 8 2}$ & 381,9 & 22,8 \\
\hline $\mathbf{1 9 6 4}$ & 9,3 & 5,8 & $\mathbf{1 9 8 3}$ & 402,9 & 22,8 \\
\hline $\mathbf{1 9 6 5}$ & 9,1 & 5,0 & $\mathbf{1 9 8 4}$ & 475,0 & 25,6 \\
\hline $\mathbf{1 9 6 6}$ & 10,6 & 5,7 & $\mathbf{1 9 8 5}$ & 402,9 & 21,8 \\
\hline $\mathbf{1 9 6 7}$ & 6,6 & 4,6 & $\mathbf{1 9 8 6}$ & 414,5 & 21,6 \\
\hline $\mathbf{1 9 6 8}$ & 4,1 & 2,7 & $\mathbf{1 9 8 7}$ & 317,7 & 17 \\
\hline $\mathbf{1 9 6 9}$ & 6,9 & 3,5 & $\mathbf{1 9 8 8}$ & & 13 \\
\hline $\mathbf{1 9 7 0}$ & 5,5 & 3,0 & $\mathbf{1 9 8 9}$ & 306 & 13 \\
\hline $\mathbf{1 9 7 1}$ & 5,0 & 2,5 & $\mathbf{1 9 9 0}$ & 285 & 10,9 \\
\hline $\mathbf{1 9 7 2}$ & 7,4 & 3,4 & $\mathbf{1 9 9 1}$ & 265 & 9,3 \\
\hline $\mathbf{1 9 7 3}$ & 14,7 & 6,1 & $\mathbf{1 9 9 2}$ & 515 & 14,7 \\
\hline $\mathbf{1 9 7 4}$ & 24,1 & 8,6 & $\mathbf{1 9 9 3}$ & 667 & 17,5 \\
\hline $\mathbf{1 9 7 5}$ & 53,3 & 14,2 & $\mathbf{1 9 9 4}$ & 699 & 16,6 \\
\hline $\mathbf{1 9 7 6}$ & 136,4 & 24,3 & $\mathbf{1 9 9 5}$ & 797 & 17,1 \\
\hline $\mathbf{1 9 7 7}$ & 154,8 & 23,4 & $\mathbf{1 9 9 6}$ & 1024 & 23,2 \\
\hline $\mathbf{1 9 7 8}$ & 159,4 & 20,4 & $\mathbf{1 9 9 7}$ & 1031,7 & 23,7 \\
\hline $\mathbf{1 9 7 9}$ & 180,4 & 19,6 & $\mathbf{1 9 9 8}$ & 947 & 20,9 \\
\hline
\end{tabular}

Source : Central Bank of Jordan

41 Cette date voit la perte, cette fois totale, des remises des travailleurs émigrés, dont la baisse était déjà ressentie depuis le milieu des années quatre-vingt mais qui finançait encore $85,5 \%$ du déficit commercial jordanien en 1989. La position perçue comme favorable à l'Irak du roi Hussein avait entraîné en 1991 un arrêt des versements de l'aide arabe à la Jordanie ${ }^{28}$. Les relations tendues avec les autres pays du Golfe affectent aussi gravement les revenus tirés des exportations (ESCWA, 1992:4) : les marchés des deux principaux partenaires commerciaux de la Jordanie, le Koweit mais aussi l'Irak ${ }^{29}$, sont perdus en raison des sanctions contre ce pays ${ }^{30}$.

42 En conséquence, des phénomènes tels que le chômage, la pauvreté, déjà en expansion depuis le début des années quatre-vingt, ont accentué leur progression. Les returnees ayant accru de $10 \%$ environ la force de travail, les taux de chômage ont dépassé les 30 \% (ESCWA, 1992) au début de la décade. Sur un marché du travail si saturé, l'épargne rapportée du Golfe par les ménages s'est épuisée rapidement. La pauvreté, qui affectait officiellement de 15 à $20 \%$ de la population au cours des années quatre-vingt, marque une hausse à 19,8 \% en $1991^{31}$ (HKJ, 1991). De plus, la pression sur les infrastructures et les ressources naturelles a été très forte, de même que sur le marché locatif et foncier, entraînant une hausse des prix qui a touché l'ensemble de la population.

Mutations radicales du contexte et des moyens d'accès au capital

Après la rupture des relations entre les deux rives du Jourdain ${ }^{32}$, une chaîne d'événements, dérivés en partie de la chute du revenu de la rente, change le contexte politique jordanien et achève l'œuvre déstabilisante du renversement des mouvements migratoires à l'égard de la redistribution des richesses, des possibilités d'accès au capital et des liens État-société. La mise en place d'un Programme d'Ajustement structurel sanctionne la faillite économique jordanienne, et remet ainsi en cause les liens État-société, fondés sur le processus de redistribution clientéliste. Cette crise 
économique prendra sa source dans le tarissement des revenus tirés de l'émigration des citoyens. En 1994, la signature du traité de paix avec Israël affectera aussi profondément les modalités de l'intégration des réfugiés palestiniens au sein de l'entité nationale jordanienne.

Nous avons montré à quel point la stabilité du régime dépendait étroitement de ses revenus, tirés des remises des travailleurs immigrés et de l'aide arabe et internationale, plus faciles à capter et à redistribuer sous forme de prébendes par le régime. Pour des raisons de stabilité politique énoncées plus haut, la logique clientéliste primait sur la rationalité économique et sur la productivité. La diminution des remises des travailleurs émigrés, alliée à celle de l'aide arabe, la récession économique régionale à partir de 1983 ont commencé dès lors à faire entrer la Jordanie dans la récession générale (Al-Saïd, 2000). Loin d'engager des mesures d'austérité, de diminuer les dépenses publiques, le gouvernement se lance dans une politique expansionniste; de nombreux prêts sont négociés auprès d'institutions financières occidentales et internationales, telles le FMI et la Banque mondiale. En 1988, la dette extérieure de la Jordanie s'élevait à huit milliards de dollars, soit $200 \%$ du PIB (Dougherty, 1996). A cette date, pour la première fois, la Jordanie n'ayant pas pu rembourser les montants prévus, le gouvernement se voit contraint de signer son premier plan à moyen terme d'ajustement structurel avec la FMI et la Banque mondiale. Ce plan prévoyait, entre autres mesures, une réforme du secteur public et l'engagement d'une politique de privatisation (Al-Saïd, 2000), une réduction des dépenses publiques (réduction des effectifs de l'Armée, allègement des subventions de l'État aux denrées de première nécessité), mais aussi l'accès à l'aide étrangère et le rééchelonnement de la dette

Le désengagement forcé de l'État par la privatisation des moyens de production et la rationalisation de l'économie bouleversent l'édifice des relations État- société soustendant la stabilité du régime hachémite, à savoir la redistribution clientéliste des ressources. De fait, le déclenchement d'émeutes dans les régions rurales de la Jordanie en avril 1989 constitue une conséquence lointaine du renversement des flux migratoires. Ces régions ont particulièrement souffert de l'ajustement structurel, en raison de leur dépendance vis-à-vis de l'État, alors contraint de contracter ses services (emplois dans le secteur gouvernemental et l'Armée, éducation, santé).

Le régime a d'ailleurs saisi toute la dimension politique de la revendication, en instaurant le " processus contrôlé de libéralisation politique, de manière à canaliser les frustrations et à partager la responsabilité politique d'autres mesures d'austérité » (Wils, $1998: 110$ ).

47 Suivant ces deux événements, intervient la signature des traités de paix avec Israël. Après les accords d'Oslo, signés le 13 septembre 1993 à Washington, entre I. Rabin et Y. Arafat, qui procurent à tous les Palestiniens une identité politique sinon encore territoriale, la ratification du traité de paix du Wadi Araba le 26 octobre 1994 pousse la Jordanie à reconnaître officiellement l'État d'Israël. Elle oblige aussi cette dernière à reconnaître comme irréalisable le choix de la Jordanie comme patrie de substitution, garantissant du même coup plus sûrement que jamais l'existence de la Jordanie hachémite.

Mais par cette décision, la Jordanie fixe également sur son territoire les réfugiés de 1948. Elle reconnaît implicitement comme impossible leur retour vers les territoires maintenant israéliens. Les déplacés quant à eux se retrouvent dans une situation ambiguë : leur "droit au retour », par le biais des négociations multilatérales sur le sort 
des réfugiés, se trouve discuté, mais au plus haut niveau politique, hors de leur contrôle. Brusquement et arbitrairement, des appartenances identitaires à référent national se trouvent définies, après avoir été niées depuis 1967. Les Jordaniens d'origine palestinienne se trouvent stigmatisés comme "Palestiniens» et non plus selon leur statut légal. Ils sont donc menacés implicitement dans leur appartenance jordanienne, puis séparés entre réfugiés, déplacés, returnees, certains, implicitement encore, appelés à rester sur le territoire, d'autres (les déplacés), potentiellement incités à rejoindre la rive ouest du Jourdain.

Migrations de retour et politique

L'analyse de l'ensemble de ces facteurs, démographiques, économico-politiques et directement politiques suggère une mutation radicale très déstabilisante des relations État-société. Les modalités de la citoyenneté, le volume des ressources disponibles et les moyens d'y accéder sont également remis en cause par les migrations de retour de la fin des années quatre-vingt. Ces mutations concernent l'ensemble de la population jordanienne. Les migrations de retour ont non seulement entraîné la faillite de l'État rentier et de la structure socio-politique qu'il soutenait, mais aussi la marginalisation de la plupart des migrants concernés. Le capital social des returnees, acquis par l'émigration n'a pas été transférable en Jordanie comme l'indiquent leurs difficultés d'insertion, autant au niveau social qu'économique.

Les structures de pouvoir héritées de l'État rentier ont par contre survécu à l'ensemble des événements politiques. Depuis 1988, l'État reste maître du processus de définition de l'identité nationale et des modalités de la citoyenneté, alors que celles de la redistribution ont été profondément modifiées. Dans le contexte d'ouverture démocratique et d'émergence d'une "sphère publique" de discussion après 1989, comment les migrants ont-ils pu participer au processus de développement politique en cours?

51 Une des caractéristiques des returnees reste leur intégration imparfaite dans le tissu social jordanien, comme le remarquent l'ensemble des enquêtes réalisées sur cette population (par exemple : Van Hear, 1994a ; b ; Radi, 1994 ; Shami, 1996 ; Le Troquer et Hommery, 1998). Ils souffrent d'une absence de capital social, mais aussi politique, mobilisables sur le sol jordanien. Les difficultés de leur incorporation économique dans les structures publiques et privées en portent une part de responsabilité. Pour la plupart des returnees, l'arrivée en Jordanie se traduit par une mobilité sociale clairement descendante ${ }^{33}$.

52 A cette frustration des returnees s'ajoute celle du reste de la population, qui voit se réduire les opportunités disponibles, ce qui entraîne une hostilité de la population jordanienne dans son ensemble vis-à-vis des returnees. Le chômage des diplômés, catégorie de la population prompte à la revendication, était estimé à $23 \%$ par le ministère du Travail en 1992 (Van Hear, 1994b), situation alimentant la rancune à l'égard des nouveaux venus. Plus généralement, la pression sur les infrastructures, l'utilisation massive des services gratuits ou subventionnés, par une population habituée à de tels privilèges au Koweit, la hausse du prix de l'immobilier, des biens de première nécessité et la chute générale du niveau de vie sous l'effet de la crise globale du début des années quatre-vingt dix ont creusé un clivage au sein de la population, dont les returnees ont été accusés par l'opinion. Le développement de la petite délinquance, né de l'expansion de la pauvreté (Ugolini, 1993), phénomène inconnu jusqu'alors, est aussi porté au discrédit des immigrants. 

le terrain jordanien qui auraient permis, au plan économique, l'établissement d'entreprises privées viables et, au plan socio-politique, une meilleure intégration. L'emploi du terme returnee laisse entendre le retour dans une patrie d'origine, où une partie de la famille a été laissée. Or, seuls $10 \%$ des premiers arrivés ont été hébergés par leurs familles (NCERD, 1991). Ceci montre les limites de la famille étendue : les liens ne résistent pas toujours à une longue absence, de même qu'ils se distendent dans la plupart des cas, si le fardeau représenté par les parents immigrés s'alourdit, en raison ici de la crise économique. Les exemples de tels scénarios de ruptures entre ménages apparentés abondent (Shami, 1996 ; Le Troquer et Hommery, 1998), ce qui a ajouté au rejet des returnees dans l'opinion et à l'isolement et l'amertume de ceux-ci. d'identification politique des immigrés du Golfe en Jordanie a été très faible, cause et conséquence de leur marginalisation dans le pays et de leur perte de capital. surtout de l'ouverture démocratique en cours depuis 1989 faisait attendre leur participation aux élections législatives de novembre 1993. Les nationalistes transjordaniens l'appréhendaient, les partisans d'une évolution politique l'attendaient avec espoir. L'allégeance allant de pair avec le bénéfice des largesses du régime, on pouvait s'attendre à ce que la population des returnees, qui n'en avait pas profité, revendique en contrepartie une participation dans le jeu politique. Pourtant, il n'en a rien été. Le seul candidat issu des returnees n'a pas été élu. La participation de ceux-ci au vote a été faible.

On voit ici que les migrations de retour, en entraînant la chute de l'État rentier, ont provoqué un bouleversement radical des modalités de l'accès au capital en Jordanie. Les zones rurales, qui détenaient jusqu'à la fin des années quatre-vingt des possibilités d'accès au capital grâce à leurs liens privilégiés avec le régime, et qui n'avaient, pour cette raison, que peu recours à l'émigration internationale, se trouvent écartées des réseaux de redistribution clientéliste de l'État. De plus, les accords d'Oslo et le traité de paix du Wadi Araba ont fait fondre les derniers espoirs d'un retour en Palestine pour les réfugiés, tandis que la rupture des relations entre les deux rives du Jourdain engage des négociations entre le régime et la population sur la définition d'une citoyenneté jordanienne. L'immigration des returnees, enfin, dont les attaches avec la Jordanie sont faibles, intervient dans ce contexte de repli des redistributions étatiques, de crise économique, de débat identitaire et de construction difficile d'une citoyenneté. On peut donc interpréter la reprise d'une émigration massive, observée justement à partir de 1994, mais aussi ses caractéristiques nouvelles que l'on décrit maintenant, comme la marque d'un échec de la transition démocratique engagée en Jordanie en 1989, d'une impossibilité d'expression politique. Plus généralement, ce mouvement peut traduire une défiance à l'égard des possibilités d'incorporation des divers secteurs de la population dans les diverses dimensions (économique, sociale et politique) de la construction nationale jordanienne, un échec du contrat social.

Faillite du contrat social et reprise de l'émigration internationale à partir de 1994

On postulera ici que la reprise de mouvements migratoires à partir de 1994, cette fois perçus comme définitifs et dirigés vers les pays occidentaux, constitue un exutoire à l'absence persistante d'institutions intermédiaires aptes à se réapproprier le processus 
de redistribution des ressources, à engager une dynamique de développement et d'accès au capital au niveau local.

Nouvelles migrations, nouveaux migrants

Outre celle du profil des migrants jordaniens, la mutation des champs migratoires au départ de ce pays reste frappante. A la date du recensement en octobre 1994, le nombre de personnes enregistrées comme returnees en Jordanie (rive est) n'était plus que 216 133 (5,2\% de la population totale), arrivées entre 1990 et 1994 . Ceci laisse supposer un mouvement de retour vers le Golfe, ou de ré-émigration vers d'autres destinations car l'on considère exacte l'estimation de 300000 returnees entrés en Jordanie entre 1990 et 1992.

La période des retours du Golfe a été en effet vite suivie des premiers symptômes d'une réelle et massive volonté d'émigrer vers les pays occidentaux et l'Australie, en raison non seulement des mutations dans la composition des marchés arabes du travail, mais surtout d'un déni de confiance politique envers ceux-ci. Dès 1991-1992, 25000 demandes de visa d'émigration avaient été déposées auprès des ambassades du Canada et d'Australie à Amman (Le Troquer et Hommery, 1998).

Le recensement de 1994 indique un effectif total de 29437 Jordaniens expatriés à l'étranger, effectif manifestement sous-évalué $e^{34}$. Une autre source plus tardive $\mathrm{e}^{35}$ avance pour la fin des années quatre-vingt dix l'estimation d'environ 800000 personnes $\left(\right.$ Zaqqa, 2001) ${ }^{36}$. Cette reprise de l'émigration à partir de 1994 est également visible à travers les données du département de la Sécurité publique, qui enregistre les mouvements aux frontières (figure 1$)^{37}$.

61 Concernant la destination des migrants, selon le recensement, $36 \%$ seulement de ceuxci résident dans un pays arabe du Moyen-Orient, la majorité vivant en Irak (23,6\% des migrants dans un pays du Moyen-Orient), en Arabie Saoudite ( $26 \%$ ), en Syrie et dans le Émirats (respectivement 13,3\% et 11,9\%). Les résidents au Koweit déclarés ne sont qu'au nombre de 690 (7,8 \% des émigrés dans les pays du Moyen-Orient). La destination favorite reste les États-Unis, $23,3 \%$ des expatriés jordaniens y résidant. Curieusement, l'effectif annuel des émigrants jordaniens vers ce pays semble être resté stable au cours des années quatre-vingt dix (environ 4500 personnes par an), mais les autorités estiment que l'immigration illégale a augmenté fortement et régulièrement, jusqu'à atteindre $70 \%$ des effectifs annuels de migrants légaux ${ }^{38}$. Une partie des migrants transite en effet par le Canada ${ }^{39}$, qui a vu le nombre de demandes de visa d'émigration lui étant adressées plus que tripler depuis $1995^{40}$.

62 Ces résultats, même si les effectifs totaux restent sous-estimés, sont cohérents avec les résultats d'une enquête sur les conditions de vie en Jordanie, menée en 1997. Les enquêtés mentionnent parmi leurs apparentés une majorité d'expatriés vers le Canada et les États-Unis, des effectifs presque similaires résidant en Arabie Saoudite. Le Koweit abrite en 1997 trois fois moins d'expatriés jordaniens que ce dernier pays (FAFO, 1998 : 91-95). Les données de la troisième source donnent plus de $50 \%$ de migrants vers l'Amérique du nord (Zaqqa, 2001).

63 Le Koweit n'a pas recruté de nouveau des Jordaniens. En 1994, devant la hausse du chômage et la crise sévissant en Jordanie, le gouvernement avait pourtant tenté de restaurer les relations avec l'Émirat. Des journaux avaient annoncé des mesures encourageant le retour de quelques dizaines de milliers d'employés jordaniens, mais 
elles n'ont pas été suivies d'effets (Le Troquer et Hommery, $1998: 62$ ), ce que confirme la stagnation des effectifs expatriés vers ce pays.

Les données disponibles fournissent aussi quelques indications sur le profil des migrants. L'enquête FAFO indique une prépondérance de jeunes hommes adultes à l'étranger, suggérant une émigration avant tout temporaire, d'étudiants ou de jeunes professionnels. Dans le Golfe, la majorité des émigrants jordaniens déclarent leur emploi comme la raison principale de leur résidence à l'étranger. Cependant, la nouveauté de ce régime migratoire ne permet pas d'affirmer que la migration est réellement temporaire, même si les personnes se déclarant comme installées à l'étranger ne sont nombreuses que parmi celles expatriées dans des pays arabes autres que les pays du Golfe (FAFO, 1997 : 95). L'enquête de N. Zaqqa indique que $72 \%$ des enquêtés de son échantillon sont accompagnés par 2,8 personnes en moyenne (époux (se) et enfants). Également, seuls $61 \%$ des migrants ont reçu au moins une partie de leur éducation hors de Jordanie, ce qui suggère l'élargissement du recrutement migratoire à des personnes qui étaient peu familiarisées avec l'étranger.

Ce dernier résultat, traitant d'un sujet encore mal connu car de déclenchement récent, rejoint toutefois les observations effectuées auprès de la population. L'élargissement du recrutement migratoire constitue une réalité, même si ce facteur n'est pas abordé dans les enquêtes ${ }^{41}$ et reste donc pour l'heure impossible à chiffrer avec précision. Outre les descendants des réfugiés et déplacés de 1948 et 1967 et les ré-émigrants rentrés du Golfe, de plus en plus de personnes originaires des régions autrefois peu concernées par les mouvements hors des frontières tentent de s'expatrier. Il s'agit des habitants des zones rurales et plus généralement, des Jordaniens ni réfugiés ni déplacés. Cette évolution peut être expliquée par la diminution des opportunités dans le secteur public et dans l'Armée depuis 1988, par le développement général de l'éducation y compris dans les zones rurales ${ }^{42}$, deux facteurs se combinant pour pousser les jeunes originaires de ces régions à rechercher un capital distinct de celui dont jouissaient leurs parents. La presse rapporte régulièrement le malaise des jeunes diplômés, l'absence d'opportunités d'emploi dans une économie en récession mais fonctionnant toujours sous le régime du clientélisme. Les faibles salaires ne compensent pas l'inflation, et la situation politique de la région au tournant $\mathrm{du} \mathrm{xxI}^{\mathrm{e}}$ siècle n'attire pas l'investissement, même de la part des Jordaniens eux-mêmes ${ }^{43}$.

Réseaux familiaux, capital migratoire

66 Les données de l'enquête sur les expatriés menée par Zaqqa suggèrent que le nouveau processus migratoire repose sur la disposition d'un capital. La migration a été l'objet d'une organisation préliminaire: $67 \%$ des migrants avaient reçu l'assurance d'un emploi ou d'une place dans une institution d'enseignement dans le pays d'accueil avant le départ. Les réseaux familiaux jouent un rôle important, et confirment également les effectifs de migrants installés à l'étranger : $72 \%$ des migrants avaient des apparentés dans le pays de destination (Zaqqa, 2001).

67 La force des réseaux familiaux en Jordanie dérive de la structure clientéliste du processus de redistribution des ressources économiques et politiques ${ }^{44}$ (Czichowski, 1988). Ce facteur va également de pair avec le modèle «néopatriarcal» (Sharabi, 1996) de gouvernement, qui, en assimilant le politique au biologique, renforce les liens au sein du groupe de parentée ${ }^{4}$. Il est également lié au développement de la rente, qui a permis à une forme d'Etat-providence de se mettre en place en Jordanie au cours des années soixante-dix, et a favorisé la stabilité des structures de pouvoir à la fois au sein 
de la société et de l'unité familiale (De Bel-Air, 2001). Cette force des réseaux familiaux en place à l'arrivée des victimes des exodes de 1948 et 1967 avait d'ailleurs constitué l'un des principaux obstacles à l'incorporation des immigrés en Jordanie. En retour, la rareté des alternatives à l'institution familiale parmi les institutions sociales avait également conservé et renforcé la cohésion du groupe familial chez les réfugiés et déplacés ${ }^{46}$.

Il semble donc que le capital disponible (en termes économiques, mais aussi de réseaux familiaux comme capital social), s'il n'a pas été mobilisé dans un processus de développement politique au niveau local, l'est pour favoriser la sortie du pays. Paradoxalement, le faible capital garanti à ses citoyens par la Jordanie, si ce n'est en termes d'éducation (capital humain) et, par défaut d'alternative, en termes de cohésion familiale (capital social), semble représenter un « capital migratoire » efficace.

Émigration et stabilité politique : « Exit, Voice and Citizenship " $^{47}$

Ce mouvement accru et ses caractéristiques trouvent leur source dans l'absence de capital disponible en Jordanie, mais vont également avoir un impact politique sur le pays. Les troubles politico-économiques des années 1989-1992 ont pu être, sinon résolus, du moins calmés. L'hégémonie persistante du régime et l'absence d'expression publique sur le territoire ont été rendus possibles par cette émigration, comme au début des années soixante-dix. C'est pourquoi le régime a réactivé, au cours des années quatre-vingt dix, certaines mesures d'encadrement des migrations interrompues par la Guerre du Golfe.

Depuis 1985 déjà, l'intérêt renouvelé du régime pour les migrants avait en effet pu être remarqué. En cette période de décrue des remises des travailleurs expatriés, la préoccupation croissante pour la question des migrations et leurs conséquences sur la Jordanie se focalisait aussi sur les moyens d'inciter les émigrés, en particulier les émigrés du Golfe, à investir en Jordanie. De 1985 à 1989, des conférences annuelles sont organisées sur le sujet à Amman par le ministère du Travail ${ }^{48}$.

71 Ces réunions sont l'occasion de rappeler la cohésion nationale jordanienne au-delà des frontières, traduite dans les représentations persistantes de la migration comme phénomène temporaire (RSS, 1983). Et ceci en dépit de la réalité de l'installation des migrants dans les pays d'accueil, en particulier au Koweit jusqu'en 1990.

72 Cette volonté de ne pas couper les liens avec la main-d'œuvre expatriée s'affirme au long des années quatre-vingt, alors que des progrès sociaux sont réalisés en Jordanie : la protection sociale (assurance maladie, cotisation retraite) garantie aux travailleurs jordaniens résidant dans le pays est officiellement étendue aux expatriés, sur la base d'affiliations volontaires (Share', 1991).

73 Après une longue interruption, les conférences reprennent. À l'été 2001, un symposium se tient sur le sujet à Amman, organisé cette fois par les plus hautes instances politiques : le bureau du roi (Diwan el-malaki). Le roi Abdallah II prononce d'ailleurs luimême le discours d'introduction ${ }^{49}$, ce qui traduit la nouvelle importance donnée aux liens entre migrants et pays de départ.

74 Parallèlement à cet intérêt manifesté par l'Etat à l'égard des émigrés, des données d'enquête signalent un désengagement des expatriés vis-à-vis de la Jordanie. Selon $\mathrm{N}$. Zaqqa, $73 \%$ des migrants participant à son enquête n'étaient pas sûrs de vouloir rentrer en Jordanie ou déclaraient ne pas le souhaiter. $61 \%$ pensaient que leur contribution au développement, lors d'un éventuel retour en Jordanie, ne serait pas la 
bienvenue, et $28 \%$ en étaient sûrs. $11 \%$ seulement s'imaginaient qu'elle le serait. Conséquence de ce pessimisme, seuls $8 \%$ des remises des émigrés avaient été utilisés comme investissement productif (sur le marché boursier ou dans une affaire) (Zaqqa, 2001).

On peut alors postuler que la surprenante stabilité du régime est à porter au crédit de ce mouvement accru. L'examen des remises des travailleurs migrants montre que celles-ci atteignent à partir de 1996 des niveaux plus élevés qu'au milieu des années quatre-vingt ${ }^{50}$ (graphique 2) et constituent de nouveau une part non négligeable du PIB (tableau 3). Les flux de la rente migratoire semblent donc avoir pu être réactivés.

Figure 2 : Remises des émigrés jordaniens, 1961-1998 Montants bruts, en millions de dinars [afficher]

Cependant, si l'on en croit les résultats de l'enquête de Zaqqa, la défiance envers les capacités de redistribution du régime et de l'avenir de la Jordanie reste profonde. De plus, le sentiment de citoyenneté de ces expatriés semble peu développé, à en croire les représentations qu'ils avancent de leur possible impact sur le développement en Jordanie. On peut donc imaginer que la migration sera, sinon définitive, au moins de longue durée, et que les émigrés n'auront qu'un faible impact sur la poursuite de l'entreprise de construction nationale, si ce n'est par le biais de la rentification de l'économie. L'encouragement implicite de l'État aux migrations traduit peut-être la poursuite d'une nouvelle source de rente, venue cette fois des pays occidentaux.

ouvelles tendances migratoires semblent donc bien constituer un signe de l'échec $\mathrm{du}$ « contrat social » en Jordanie, le prix de la stabilité politique du pays. La sortie (exit), compense l'impossibilité d'expression publique (voice) et le sentiment général de difficulté de construction d'une citoyenneté. Elle indique l'amertume ressentie devant le manque d'opportunités (économiques et politiques) disponibles, alors que l'État se rentiérise de nouveau. On devra donc encore attendre, pour savoir si les tentatives récemment engagées pour rapprocher les migrants de ce dernier auront un impact au niveau politique.

Après le 11 septembre 2001 : migrations, sécurité, conséquences sur les pays de départ

A la fin des années quatre-vingt dix, la mutation des champs migratoires consécutive à la quasi-fermeture des marchés du Golfe, le développement de réseaux englobant des migrants originaires de régions auparavant peu touchées par des migrations de longue durée vers les pays occidentaux avaient permis la mobilisation d'un nombre croissant de migrants, dont les remises avaient atteint des montants comparables (en proportion du PIB) à ceux du début des années quatre-vingt. On avait suggéré que ce mouvement jouait de nouveau le rôle qu'il avait occupé durant les années soixante-dix.

Mutation des politiques des pays d'accueil ?51

Pourtant, ce mouvement est tributaire des politiques d'immigration mises en place par les pays d'accueil. Or, depuis les événements du 11 septembre 2001, le lien entre immigration et sécurité y a été mis en relief de façon appuyée. Les politiques d'immigration massives mises en place au Canada par exemple ont été critiquées, tant dans ce pays qu'aux États-Unis ${ }^{52}$ (Adelman, 2002). Un débat s'est engagé sur le sujet et sur l'intégration culturelle, et non plus seulement économique, des communautés immigrées dans les pays d'accueil. La théorie du « choc des civilisations » a été évoquée, traduisant la perception d'un échec des politiques migratoires passées, mais aussi 
l'instrumentalisation accrue du phénomène migratoire dans le discours politique (Faist, 2002).

Pourtant, les politiques migratoires de ces deux pays, qui reçoivent comme on l'a vu un nombre croissant d'immigrants arabes et moyen-orientaux, n'ont pas, à ce jour, été profondément remaniées ${ }^{53}$. Aussi les effectifs de migrants (immigrants économiques et demandeurs d'asile) en provenance du Moyen-Orient ont poursuivi leur progression jusqu'à nos jours, tant au Canada qu'aux États-Unis (Adelman, 2002 ; Camarota, 2002). Dans ce dernier pays, le nombre d'immigrants moyen-orientaux a été multiplié par sept entre 1970 et 2000, passant de moins de 200000 personnes à 1,5 million, dont environ $10 \%$ seraient installés illégalement. Sans changement de la politique américaine d'immigration, 1,1 million supplémentaire d'immigrants moyen-orientaux sont attendus sur le territoire d'ici 2010. Le 11 septembre n'a pas fait fléchir le volume des candidatures: en octobre 2001, le Département d'État aurait reçu 1,5 millions de candidatures de cette région ${ }^{54}$ à la loterie des visas ${ }^{55}$ (Camarota, 2002).

Migrations et sécurité nationale

Or, c'est justement la relative stabilité de ces chiffres qui pose problème. La progression des effectifs de migrants est plus lente que celle des demandes, dont l'augmentation au cours des années quatre-vingt dix a été très forte. Le ralentissement des procédures d'admission en résultant, les exigences accrues posées aux candidats, en termes de capital (formation et niveau d'études en particulier), risquent d'affecter le rôle des migrations comme exutoire au chômage et à l'absence de possibilités de participation politique. On peut donc se demander quelle réponse les États moyen-orientaux, où la transition démocratique attendue ne s'est pas produite, vont pouvoir fournir à ces difficultés croissantes d'exporter la main-d'œuvre surnuméraire et la contestation politique.

À cette situation se superpose l'éventualité de mouvements de population vers la Jordanie, qui ajouteraient à la pression démographique sur les ressources. Le contexte troublé de la seconde Intifada, déclenchée en septembre 2000, voit la résurgence de rumeurs touchant à un transfert de la population palestinienne vers la Jordanie. Cette situation a réveillé les craintes des nationalistes jordaniens, mais semble confirmer la tendance à une "nationalisation" de la politique jordanienne. La presse jordanienne, depuis la fin 2001, se fait régulièrement l'écho de la fermeture des frontières aux Palestiniens souhaitant fuir, même temporairement, leur lieu de résidence ${ }^{56}$. De fait, les visiteurs en provenance de Cisjordanie sont contrôlés minutieusement, la durée de leur séjour est limitée ${ }^{57}$. On ne dispose pas d'estimation fiable du nombre de Palestiniens ayant traversé le fleuve depuis le début du conflit, mais les autorités jordaniennes mettent en relief les mouvements de retour vers la Cisjordanie en provenance de la rive est $^{58}$.

83 Les réfugiés d'Irak constituent une autre composante importante de la population présente au sein des frontières, mais dont l'impact sur le processus de construction nationale est radicalement différent. Les Irakiens, entrés en Jordanie depuis la guerre du Golfe, ne peuvent en effet prétendre à l'asile politique, donc à une installation dans ce pays, qui n'a pas signé la Convention de Genève. Ils sont considérés comme des migrants en transit vers un pays tiers par les autorités jordaniennes, qui appliquent à leur endroit une politique qualifiée de « semi-protectionniste " (Chatelard, 2002) ${ }^{59}$. Les estimations des effectifs d'Irakiens en Jordanie à la fin des années quatre-vingt dix, fournies par les autorités locales et les ONG ne sont pas fondées sur des comptages 
précis et varient de 50000 à 300000 personnes. Parmi eux, 20000 seulement disposeraient d'un titre de séjour d'un an renouvelable, obtenu après la signature d'un contrat de travail légal. Le flux d'immigration en provenance d'Irak reste constant depuis la fin de la guerre du Golfe, de même que le rythme des sorties de Jordanie. Cependant, la plupart des migrants irakiens réside ou travaille en Jordanie illégalement, pour convenance personnelle (migrations de travail pendulaires entre les deux pays) mais surtout par contrainte, en raison de l'impossibilité de régulariser le séjour ${ }^{60}$ comme d'émigrer rapidement vers un pays tiers (par une demande d'asile politique déposée au Haut Commissariat aux Réfugiés, par un recours aux filières développées par les Églises chrétiennes, réservé aux chrétiens, par l'utilisation de filières clandestines, onéreuses et peu sûres). La présence irakienne, essentiellement clandestine et perçue comme transitoire, reste donc confinée hors de l'espace social jordanien. Elle est ignorée dans le débat public et ne semble pas constituer un enjeu dans le processus de construction nationale, à la différence de celle des réfugiés de Palestine, présents ou à-venir. Les autorités jordaniennes minimisent la présence des réfugiés d'Irak sur le sol national et ont de nouveau réaffirmé officiellement la fermeture des frontières aux réfugiés du nouveau conflit de 2003. Pourtant, il reste peu probable que la situation politique et économique de l'Irak se résolve très rapidement et autorise un retour massif de tous les expatriés. Les Irakiens pourraient donc faire leur entrée dans le débat sur la citoyenneté jordanienne et le partage des ressources économiques et politiques.

Migration et développement

Pour conclure cet exposé sur le rôle joué par les migrations dans le contrôle de la pression démographique sur les ressources et l'accès au capital, une remarque s'impose : il semblerait que la politique d'exportation de la contestation, si elle a résolu à court terme les conflits, s'avère finalement préjudiciable au processus de développement global, sur le long terme, des pays de la région.

Le niveau de développement humain, dans l'ensemble des pays arabes, a fait l'objet d'un rapport alarmant des Nations-Unies (UNDP, 2002). Malgré un niveau de revenu per capita ${ }^{61}$ dans le monde arabe supérieur à celui rencontré dans d'autres régions (Asie du Sud, Afrique subsaharienne), les indices de développement humain (Human Development Index (HDI) et Alternative Human Development Index (AHDI)), qui incluent des évaluation des capacités humaines disponibles, des libertés civiles, de la justice sociale, y montrent les scores les plus bas au monde. Le pays arabe détenant le meilleur AHDI, qui se trouve être la Jordanie, ne figure qu'en $68^{\mathrm{e}}$ position, sur un total de 111 pays (données de 1998). On peut donc s'interroger sur cette spécificité du monde arabe, qui allie une persistante pauvretééc ${ }^{62}$, en termes de développement dans son sens le plus large, à des revenus moyens élevés, voire très élevés pour les États producteurs d'hydrocarbures.

On a vu l'importance du facteur de la rentiérisation de l'État et de l'économie dans le processus de négociation du lien social et des relations État/société particulier au Moyen-Orient. Cette région abrite les pays producteurs de matière première, la maind'œuvre à même de l'exploiter, mais aussi reste, des années 1950-60 à aujourd'hui, une région canalisant d'importants flux d'aide internationale en provenance des pays occidentaux. On peut donc émettre l'hypothèse d'un lien de cause à effet entre la persistance de ce système économico-politique et celle du sous-développement humain dans ces régions. Cette hypothèse permet d'écarter les causalités religieuses, voire 
culturelles, à ce dernier facteur, souvent invoquées depuis le 11 septembre. Elle privilégie des facteurs explicatifs de nature politico-économique. En outre, le niveau politique à envisager ne serait plus un ensemble (perçu comme) culturel, supranational, mais bien celui de l'État, sinon de l'État-nation. Le refus, ou les difficultés de bâtir une citoyenneté, une identité nationale sont en effet à la source de l'échec du développement humain dans le monde arabe, mais plus particulièrement moyenoriental. L'évitement de ce processus et de ses ajustements politiques a en effet été rendu possible par la rentiérisation de l'État et de l'économie, dont la cause, mais aussi la conséquence, restent les mouvements migratoires. Les difficultés nouvelles d'exportation de la contestation, en même temps que celle des cerveaux, par le recours aux migrations internationales pourraient donc devenir le point de départ d'un processus global de réappropriation des ressources, humaines et autres, de revendication des outils du développement jusqu'à présent confisqués par les régimes en place dans les États de la région.

Conclusion

On a voulu, dans cette étude, mettre en relief l'importance décisive des mouvements d'émigration internationale dans le processus de stabilisation politique en Jordanie. A deux périodes de crise, nées de flux massifs de population vers la Jordanie (1967 et 1990-1991), qui toutes deux menacent la suprématie démographique de la base de soutien du régime dans la population totale, répondent deux vagues d'émigration internationale, qui permettent d'éviter au régime une renégociation des liens de redistribution clientéliste des ressources et des modalités d'accès au capital. Les migrations sont donc un facteur constitutif du politique, en Jordanie comme dans d'autres pays arabes. A la suite de la Guerre du Golfe, la rupture des liens entre la Jordanie et les riches pétromonarchies détourne les flux migratoires du monde arabe vers l'occident. On peut remarquer que ces mouvements n'ont pas donné lieu à une diffusion, vers le pays de départ, de nouveaux modes de relation État-société. La demande d'émigration s'accentue, alors que l'offre n'augmente pas, voire se réduirait en cas de changement de politique migratoire, comme le laissent présager les événements du 11 septembre. Le verrouillage politique de plus en plus marqué dans les pays arabes laisse attendre une transition démocratique troublée si elle peut se réaliser en profondeur, par une réappropriation des ressources humaines auparavant évincées du processus de construction nationale et de développement par l'émigration.

ADELMAN Howard (2002) Canadian Borders and Immigration Post 9/11, International Migration Review, vol. XXXVI, 1, pp. 15-28.

AHMED I. (1997) Exit, Voice and Citizenship, in Tomas Hammar, G. Brochmann, K. Tamas et Thomas Faist Eds., International Migration, Immobility and Development. Multidisciplinary Perspectives, Oxford / New York, Berg, pp. 159-185.

Al-SAID Hamed (2000) Jordan: The Political Economy of Public Sector Growth and Reform in a Rent-Seeking Economy, 1973-1998, Document du CERMOC n9, 16 p.

91 ANDERER G. (1991) Die Politische Ökonomie eines Allokationssystems- Jordanien und die Internationale Arbeitsmigration seit 1973, Frankfurt am Main/ Bern/ New York/ Paris, Lang.

92 AUGÉ Jean-Christophe (1997a) La privatisation en Jordanie : mise en perspective et état des lieux, Jordanies, n²4, décembre 1997, pp. 131-146. 
AUGÉ Jean-Christophe (1997b) Système d'enseignement supérieur et populations étudiantes en Jordanie : pistes de recherche, Jordanies, n³, juin 1997, pp. 127-133.

BEBLAWI Hazem (1987) The Rentier State in the Arab World, in Hazem Beblawi et Giacomo Luciani Eds., The Rentier State, in Nation, State and Integration in the Arab World (vol. 2), London/ New York, Croom Helm.

BRAND Laurie (1988) Palestinians in the Arab World. Institution Building and the Search for State, New York, Columbia University Press.

BRAND Laurie (1995) Jordan's Inter-Arab Relations: The Political Economy of Alliance Making, New York, Columbia University Press.

CAMAROTA Steven (2002) The Muslim Wave. Dealing with Immigration from the Middle East, National Review, August 2002, http://www.cis.org/articles/2002/ sac830.htm

CHATELARD Géraldine (2002) Jordan as a Transit Country: Semi-Protectionist Immigration Policies and Their Effect on Iraqi Forced Migrants, Communication présentée à l'atelier $\mathrm{n}^{\circ}$ 14, Third Mediterranean Social and Political Research Meeting, Montecatini Terme and Florence : Robert Schuman Centre for Advanced Studies, 20-24 mars 2002.

CZICHOWSKI Frank (1988) 'Ich und meine Vettern gegen die Welt...' Migration, 'Wastah', Verteilungskoalitionen und gesellschaftliche Stabilität in Jordanien, Orient 29, n 4, décembre 1988, pp. 561-569.

CZICHOWSKI Frank (1990) Jordanien: Internationale Migration, Wirtschaftliche Entwicklung und Soziale Stabilität, Hamburg, Deutsches Orient-Institut (Schriften des Deutschen Orient-Institut).

1 DE BEL-AIR Françoise (2001) From household politics to domestic policies : The effect of demographic transition on socio-political patterns of domination, in George Joffe Ed., Jordan in Transition- 1990-2000, London, Hurst, pp. 149-171.

DIB Georges (1979a) Legal Aspects of Intra-Regional Migration: Proposal for Action, Paper presented at the Second Regional Population Conference of ECWA, Damascus, ECWA, 1-6 December 1979.

DIB Georges (1979b) Migration and Naturalization Laws in Egypt, Lebanon, Syria, Jordan, Kuwait and the United Arab Emirates, Part I : Migration Laws, Population Bulletin of ECWA, $\mathrm{n}^{\circ} 15$.

DOUGHERTY Pamela (1996) Les émeutes du pain à Kérak: une réponse au processus d'ajustement structurel en cours, Jordanies, n², décembre 1996, pp. 95-99.

5 ESCWA (1992) Proceedings - Expert Group Meeting on the Absorption of Returnees in the ESCWA Region with Special Emphasis on Opportunities in the Industrial Sector, Amman, 16-17 December 1991.

FAFO (1997) Jordan Living Condition Survey 1996- Main Results (Tables), Amman, Norway Institute for Social Sciences (FAFO)/ Department of Statistics.

7 FAIST Thomas (1997) The Crucial Meso-Level, in Tomas Hammar, G. Brochmann, K. Tamas et Thomas Faist Eds., International Migration, Immobility and Development. Multidisciplinary Perspectives, Oxford / New York, Berg, pp. 187-217.

FAIST Thomas (2002) 'Extension du domaine de la lutte' : International Migration and Security Before and After September 11, 2001, International Migration Review, vol. XXXVI, 1, pp. 7-14. 

Statistics. Jordanie, Amman, Department of Statistics (en arabe). Population Characteristics, Amman, Department of Statistics. Economic and Social Development 1981-1985, Amman.

LAVERGNE Marc (1996) La Jordanie, Paris, Karthala. Affari Internazionali / Kent, Croom Helm. NCERD, (3 tomes) (en arabe). the Modern Arab World, London, Quartet Books, pp. 85-104. avril-juin 1994, pp. 55-65.

FARGUES Philippe (1991) La migration obéit-elle à la conjoncture pétrolière dans le Golfe ?- L'exemple du Koweit, in Gilbert Beaugé et Friedmann Buttner Eds., Les migrations dans le monde arabe, Aix en Provence, IREMAM/ Paris, CNRS, pp. 41-68.

FEILER Gil (1993) Labour Migration in the Middle-East Following the Iraqi Invasion of Kuwait, in Israël / Palestine Centre for Research and Information (IPCRI) Israël/ Palestine, Issues in Conflict, Issues for Cooperation, Jerusalem, IPCRI, vol. II, nº 7, December 1993.

FINDLAY Allan et SAMHA Musa (1985) The Impact of International Migration on the Urban Structure of Amman, Espace, Populations, Sociétés, 1, pp. 93-98.

GOROKHOFF Philippe (1985) Les Palestiniens au Koweit, in CERMOC (collectif) Migrations et changements sociaux dans l'Orient arabe, Beyrouth, CERMOC, pp. 37-84.

HASHEMITE KINGDOM OF JORDAN(1964) Population and Housing Census 1961- Volume IFinal Tables- General Characteristics of the Population, Amman, Department of

HASHEMITE KINGDOM OF JORDAN (1983) Population and Housing Census 1979 vol. 2, part 1 : Population Characteristics, Amman, Department of Statistics.

HASHEMITE KINGDOM OF JORDAN (1986) Étude sur les migrations internes, de retour et la force de travail (résultats principaux), Amman, Department of Statistics (en arabe).

HASHEMITE KINGDOM OF JORDAN (1991) Travailleurs, chômage, immigrés et pauvreté en

HASHEMITE KINGDOM OF JORDAN (1993) Enquête Les Jordaniens de l'extérieur, rentrés en Jordanie entre le 31/12/1991 et le 10/8/1992, Amman, Department of Statistics (en arabe).

HASHEMITE KINGDOM OF JORDAN (1997) Population and Housing Census 1994 vol. 2 :

HASHEMITE KINGDOM OF JORDAN / National Planning Council (1981) Five Year Plan For

LE TROQUER Yann et HOMMERY Rozenn (1998) Du Koweit à la Jordanie, le retour suspendu des Palestiniens, Revue d'Études palestiniennes, n¹4, hiver 1998, pp. 54-70.

LOEW G. (1978) L'essor urbain en Jordanie orientale, Maghreb Machrek, vol. 81, pp. 50-55.

LONGUENESSE Elisabeth (1991) Rapports de classes, solidarités communautaires et identités nationales dans les pays du Golfe, in Gilbert Beaugé et F. Buttner Eds., Les migrations dans le monde arabe, Aix en Provence, IREMAM/ Paris, CNRS, pp. 123-136.

LUCIANI Giacomo et SALAME Ghassan Eds. (1988) The Politics of Arab Integration, Istituto

NATIONAL CENTER FOR EDUCATIONAL RESEARCH AND DEVELOPMENT (NCERD) (1991) Étude sur les caractéristiques sociales et économiques des Jordaniens revenus du Golfe, Amman,

6 OWEN Roger (1983) Government and Economy in Jordan: Progress, Problems and Prospects, in Patrick Seale Ed., The Shaping of an Arab Statesman : Abd al-Hamid Sharaf and

7 RADI Lamia (1994) Les Palestiniens du Koweit en Jordanie, Maghreb-Machrek, $n^{\circ}$ 144, 
ROYAL SCIENTIFIC SOCIETY (R.S.S.) (1983) Workers Migration Abroad: Socio-Economic Implications for Households in Jordan, Amman, R.S.S. / Economic Department.

RUSSEL Sharon Stanton (1988) Migration and Political Integration in the Arab World, in Giacomo Luciani et Ghassan Salamé Eds., The Politics of Arab Integration, Istituto Affari Internazionali / Kent, Croom Helm, pp. 183-210.

RUSSEL Sharon Stanton (1992) International Migration and Political Turmoil in the Middle-East, Population and Development Review, vol. 18, n², December 1992, pp. 719-727.

SALIBI Kamal (1996) Histoire de la Jordanie, Paris, Naufal.

SAMHA Musa (1990) The Impact of Migratory Flows on Population Changes in Jordan : A Middle Eastern Case Study, International Migration, vol. XXVIII, n² 2, June 1990, pp. 215-227.

SAMHA Musa (1999) Migration in Jordan: Patterns and Impact on Population Changes, Communication présentée au colloque Migration and Culture Contact: Patterns of Confrontation and Coexistence in a Changing World, Amman, The Royal Institute for Inter-Faith Studies, 11-14 October 1999.

SECCOMBE Ian (1987) Labour Emigration Policies and Economic Development in Jordan : From Unemployment to Labour Shortage, in Bishara Khader et Adnan Badran Eds., The Economic Development of Jordan, Louvain, CERMAC / London, Croom Helm, pp. 118-132.

SHAMI Seteney (1996) Emigration Dynamics in Jordan, Palestine and Lebanon, Geneva, IOM / UNFPA, Policy Workshop on Emigration Dynamics in the Arab Region.

SHARABI Hisham (1996) Le néopatriarcat, Paris, Mercure de France.

SHARE' M. (1991) La main-d'oeuvre jordanienne dans les pays du Golfe arabe : volume, mouvements, ses régulations, son avenir, in N. Khouri et Q.A. Ahmad Eds., Structures de la main-d'oeuvre sur le marché du travail jordanien, Amman, R.S.S. / B.I.T. (bureau régional), en arabe.

SHARE' M. (1987) The Use of Jordanian Workers Remittances, in Bishara Khader et Adnan Badran Eds., The Economic Development of Jordan, Louvain, CERMAC / London, Croom Helm, pp. 32-44.

SINGER Colin (2002) http ://www.immigration.ca/permres-faq-english.asp

TELL Tareq (1994) Les origines sociales de la glasnost jordanienne, in Riccardo Bocco et Mohammed Reza Djalili Eds., Moyen-Orient : migrations, démocratisation, médiations, Paris, PUF, pp. 201-219.

UGOLINI Laetitia (1993) La justice et les mineurs en Jordanie, Rapport de stage extérieur, Bordeaux, Ecole nationale de la Magistrature.

UNDP (2002) Arab Human Development Report, New York, UNDP.

VAN HEAR Nicholas (1994a) L'impact des rapatriements forcés vers la Jordanie et le Yémen pendant la crise du Golfe, in Riccardo Bocco et Mohammed Reza Djalili Eds., Moyen-Orient : migrations, démocratisation, médiations, Paris, PUF, pp. 101-116.

VAN HEAR Nicholas (1994b) The Impact of the Involuntary Mass 'Return' to Jordan in the Wake of the Gulf Crisis, International Migration Review, vol. XXIX, n², pp. 352-374. 
WILS Oliver (1998) L'aide étrangère et son impact sur l'économie politique jordanienne : quelques questions de recherche, Jordanies, n5-6, juin-décembre 1998, pp. $100-120$.

WORLD BANK (1994) Peace and the Jordanian Economy, Washington, IBRD/ The World Bank.

WORLD BANK (1996) Jordan. Country Overview, http ://www.worldbank.org/html/ext dr/ offrep/mena/Jordan.htm

ZAQQA Nadim (2001) Jordanian Migration, http://www.jordanian-migration.com/ index.html

\section{NOTES}

1. - Cet aspect du rôle des migrations internationales sera abordé dans une étude sur les politiques d'immigration en Jordanie, publiée dans le cadre du programme « Migrations au Moyen-Orient arabe : politiques migratoires, réseaux et communautés dans le contexte de la mondialisation ", mené par l'Institut français du Proche-Orient à Amman (Jordanie).

2. -Migrants de nationalité jordanienne chassés des pays du Golfe vers la Jordanie par le conflit consécutif à l'invasion du Koweit par l'Irak en 1990 et la seconde Guerre du Golfe.

3. -Plus d'un demi-million de ces derniers étaient des réfugiés, vivant pour la plupart dans les camps : environ 400000 parmi les Palestiniens de Cisjordanie, et 100000 parmi les Transjordaniens de la rive orientale. Selon la définition de l'UNRWA (Office de secours et de travaux des Nations unies pour les réfugiés de Palestine au MoyenOrient), agence des Nations-unies créée en mai 1950 pour venir en aide aux personnes expulsées de Palestine, un réfugié palestinien est une personne qui a eu sa résidence normale en Palestine pendant deux ans au moins avant le conflit de 1948 et qui, en raison de ce conflit, a perdu à la fois son foyer et ses moyens d'existence, et a trouvé refuge, en 1948, dans l'un des pays où l'UNRWA assure des secours.

4. -Sous administration égyptienne depuis 1949.

5. -Les déplacés non réfugiés ne relèvent pas de la responsabilité de l'UNRWA, mais de celle du gouvernement jordanien, en tant que citoyens de ce pays déplacés à l'intérieur des frontières nationales d'alors, la rive ouest ayant été annexée par la Jordanie entre 1950 et 1967. La nationalité jordanienne a été en effet octroyée à tous les habitants de la Cisjordanie et aux réfugiés palestiniens incorporés dans les nouvelles frontières $\mathrm{du}$ royaume (et même à ceux résidant ailleurs, s'ils en faisaient la demande).

6. -La ville de Amman, par exemple, passe de 30000 habitants en 1943 à 615000 en 1967 (Loew, 1978).

7. -En 1948, les Palestiniens vivant en situation « précaire » constituaient déjà $37 \%$ de la population de Amman, $26 \%$ de celle d'Irbid (Loew, 1978).

8. -Même si le PIB croît en moyenne de 8 \% par an jusqu'en 1966 (Lavergne, 1996).

9. -Ce système clientéliste lie la dynastie hachémite aux principaux groupes tribaux des régions rurales et steppiques, par un contrat d'» allégeance contre protection » (Tell, 
1994), de même qu'aux grands commerçants et aux bureaucrates originaires des pays voisins, qui assuraient depuis la création de l'Emirat son encadrement économique et politique.

10. -Ce transfert de capital était plus aisé pour les membres des " grandes familles » palestiniennes, dont la plupart entretenaient déjà des liens privilégiés avec la dynastie hachémite avant 1948.

11. -Les plus touchés par le chômage jusqu'à nos jours, malgré l'investissement réalisé et le capital humain qu'ils représentent.

12. -Avec par exemple la création de l'OLP en 1964.

13. -Les effectifs de réfugiés sont publiés par l'UNRWA, mais ceux des déplacés n'ont pas été enregistrés, car on a vu que ces personnes, se déplaçant d'une rive à l'autre du Jourdain, étaient considérées comme circulant à l'intérieur d'un même pays en raison de l'annexion par la Jordanie de la rive ouest (Cisjordanie), depuis 1950.

14. -Selon des données collectées élaborées lors de la préparation aux négociations multilatérales sur les réfugiés en 1991.

15. -De la perte de la Cisjordanie envahie par Israël en 1967, aux événements de «Septembre noir » en 1971, point culminant d'un conflit mené sur le territoire jordanien, entre les organisations palestiniennes à la recherche d'une base de combat pour la libération de la Palestine et le régime hachémite.

16. -Ces données sont compilées par Zaqqa, 2001. Elles ne sont qu'imparfaites pour étudier les migrations, car elles ne distinguent pas entre tous les mouvements aux frontières (travail, tourisme, etc.). Elles restent donc essentiellement indicatives. 17. -Constitution jordanienne de 1952, articles 9-i et 9-ii.

18. -Voir par exemple pour le Koweit, Russel et Al-Ramadhan, 1994 ; Russel, 1988. Pour plusieurs autres pays du Moyen-Orient, quelques indications dans Dib, 1979a et b. 19. -À la différence des pays occidentaux, où l'on considérait la migration comme définitive, ce qui reste à vérifier (Shami, 1996).

20. -Le nombre de détachements du ministère de l'Education vers les pays du Golfe croît de 271 en 1970, 810 en 1974 à 2330 en 1983 (Seccombe, 1987 : 122).

21. -L'enregistrement des expatriés repose sur des déclarations volontaires, en accord avec la politique officielle jordanienne vis-à-vis de l'émigration. Les pays de destination n'enregistrent pas tous de la même façon (s'ils les enregistrent) les entrées et sorties de migrants : l'Irak par exemple ne détaille pas par nationalité les immigrants arabes, tandis que le Koweit, dans ses statistiques de population, agrège Jordaniens et Palestiniens sans mention du lieu de départ (rive est ou rive ouest du Jourdain). 22. -Ce chiffre émane des autorités koweitiennes, même si, de 1965 à 1970, les statistiques officielles ne distinguaient pas entre Palestiniens et Jordaniens (Brand, 1988 : 115 (note 26).

23. -Dans un article important, H. Beblawi (Beblawi, 1987) revient sur le concept de rente, de nouveau en vogue après 1973. Rétribution d'une ressource naturelle (agricole, minière...), la rente a été distinguée très tôt par les économistes classiques (Smith et surtout Ricardo) des autres formes de revenu (salaires et profits), dans le sens où elle ne constitue jamais, elle, que le reflet et non la cause du prix des produits. Et cette distinction analytique de recouper la stigmatisation sociale bien connue (commune à Marx, Weber...) du rentier comme agent bénéficiaire d'une production à laquelle il n'a pas pris une part active. De manière générale, souligne donc $\mathrm{H}$. Beblawi, c'est faute de concepts plus adéquats qu'il convient de parler d'État rentier lorsqu'on évoque la prééminence du facteur pétrolier dans les économies arabes contemporaines. Et 
l'auteur d'insister sur l'importance, en l'espèce, du caractère externe de la rente, une rente interne supposant, pour être pérenne, l'existence d'un fort secteur productif national (Augé, 1997a : 145 (note 2). J-C. Augé ajoute : « Pratiquement dépourvue d'hydrocarbures, la Jordanie [...] constitue [...] pour H. Beblawi, G. Luciani, R. Brynen entre autres [....] le cas-type d'État (semi)rentier » (Augé, 1997a : 144).

24. -Dès 1921, à la création de l'Émirat de Transjordanie, le régime vivait des allocations de la puissance mandataire britannique. Ces allocations de la Grande-Bretagne se poursuivent après 1946, mais sont progressivement remplacées, à partir de 1957, par celles des États-Unis, constituant une véritable stratégie de la part du régime.

25. -Cette théorie remet en cause les travaux inspirés des théories néoclassiques. La rente apparaît, non seulement comme un concept d'économie politique, suffisanteinsuffisante en fonction des demandes, mais comme remplissant une fonction, déterminante pour la structure politique du pays. L'argumentation théorique sur l'État rentier au Moyen-Orient, développée, en particulier, dans certaines universités allemandes, se distingue par la prise en compte attentive de facteurs autres que purement économiques ou politiques, des concepts de sociologie politique : le patronage, la notion de réseau d'influence et de groupe de pression, par exemple (Anderer, 1991).

26. -Koweit, Émirats arabes unis, Qatar, Bahrein, Arabie Saoudite.

27. -De 1989 à 1996, la part des remises des Jordaniens expatriés dans le PIB est calculée à partir des montants des remises et du PIB présentés par Wils, 1998 : tableau 3. Ces montants, qui restent indicatifs, sont cohérents avec les chiffres présentés dans les annuaires statistiques à partir de 1998 et en reflètent les fluctuations. Pour 1997 et 1998, les chiffres des remises (nettes) et de leur part dans le PIB sont tirés des Annuaires statistiques des années considérées.

28. -A l'origine, les soutiens financiers devaient s'élever à 616,1 millions de dollars par an, dont $35 \%$ à la charge du seul Koweit (Radi, 1994 ; ESCWA, 1992).

29. -Le Koweit et l'Irak absorbaient $70 \%$ de la production agricole jordanienne (Lavergne, 1996 : 148).

30. -Résolution 611 des Nations-unies décrétant l'embargo sur l'Irak.

31. -Proportion de la population en-deça du seuil de pauvreté défini par la Banque mondiale, soit un revenu per-capita inférieur à 370 \$ par an (\$ de 1985, réajusté en fonction de l'évolution du pouvoir d'achat et de l'inflation) (Worzala, $1994: 7$ ).

32. -Décision annoncée le 31 juillet 1988, donnant lieu au désengagement administratif et juridique de la Jordanie sur la rive ouest (Cisjordanie), liens qui subsistaient malgré l'invasion de la Cisjordanie par Israël en 1967. Elle fait revenir la Jordanie dans ses frontières d'avant 1950, et revêt une importance décisive, car elle ouvre la voie à la revendication, sinon à la création d'un État palestinien sur la rive ouest du Jourdain. Dans ce contexte, elle remet en cause les frontières du territoire, la place de la Jordanie dans le jeu politique régional, la signification de la nationalité et les paramètres de la citoyenneté. La population résidente sur la rive ouest se trouve de facto désignée comme palestinienne.

33. -Les personnes ayant réussi à s'insérer sur le marché du travail à un niveau équivalent à celui occupé au Koweit insistaient sur la baisse de leur niveau de vie moyen, en raison de la faiblesse des salaires jordaniens par rapport au coût de la vie. 34. -Les données concernant ces personnes ont été tirées de l'enquête sur les ménages et leurs membres, à l'aide des renseignements contenus dans les livrets de famille et 
des questionnaires adressés aux chefs de famille, comprenant des questions sur les membres du ménage résidant à l'étranger (HKJ, 1997 : III).

35. -Enquête réalisée sur Internet, adressée aux Jordaniens expatriés et aux résidents en Jordanie (Zaqqa, 2001). Le nombre de personnes ayant répondu au questionnaire n'est pas mentionné.

36. -Celle proposée par Sinclair pour l'année 1987. L'auteur indique que cette estimation est aussi utilisée par le département des Statistiques jordanien.

37. -Les entrées observées en 1994 signalent le retour de beaucoup d'expatriés d'autres régions du monde, en raison des espoirs suscités par la signature des accords d'Oslo et du Wadi Araba. Il est probable que, parallèlement, d'autres Jordaniens aient migré hors du pays au même moment.

38. -D’après un interview du consul général des États-Unis à Amman, réalisé en 1999, www.acsamman.edu.jo/ ms/immigration/atf/maloninterview.html

39. -Le Canada, d'après de nombreux témoignages, constitue la porte d'entrée sur le continent américain.

40. -Dans l'ensemble de la région (Liban, Syrie, Palestine, Jordanie), ce qui a entraîné un changement dans la procédure des demandes (entretiens avec des candidats jordaniens à la migration).

41. - Pour des raisons touchant à la cohésion nationale, facteur toujours très sensible.

42. -En 2000, la Jordanie comptait une vingtaine d'universités pour 5 millions

d'habitants, dont huit universités publiques. Le pays compte d'autre part une proportion de $28 \%$ des classes d'âges 18-23 ans inscrits dans un établissement d'enseignement supérieur, un maximum régional (à égalité avec le Liban), et « une moyenne honorable au regard de celle des pays plus riches » (Augé, $1997: 127)$.

43. -Par exemple : The Star, $2 / 07 / 98 ; 16 / 08 / 98$. Les dividendes de la paix avec Israël sont reconnus comme inexistants par la grande partie de la population, malgré l'espoir que la signature des traités avait engendré, au moins au niveau économique. Cet espoir était traduit par le grand nombre de retours observés en 1994 vers la Jordanie et la Palestine.

44. -En empêchant le développement d'une économie productive, propre à favoriser l'émergence de nouvelles classes ou groupes de pression.

45. -La référence à la parenté est omniprésente dans le discours politique jordanien. En effet, le référent familial met en relief l'unité supposée régner au sein de la collectivité nationale, voire du groupe national selon la force du lien politique le soudant. L'unité et la solidarité liant les membre de la famille jordanienne prévient aussi de façon implicite toute tentative de déstabilisation, de traitement différentiel au sein de la fratrie, à savoir, entre Jordaniens « de souche » et Jordaniens d'origine palestinienne. L'unité familiale sert donc de modèle à l'unité nationale, autre thème crucial du discours politique jordanien.

46. -L'ensemble des enquêtes menées en Palestine mentionne d'ailleurs l'importance prépondérante des liens familiaux par rapport aux autres formes de lien social au sein de la société palestinienne à travers son histoire.

47. -Ahmed, 1997. Cet article relie les migrations au politique en opposant la solution du départ à celle de l'expression publique, en cas de désaccord entre le régime et les citoyens. Le projet national, et donc la définition de la citoyenneté, interviennent alors dans la décision de migrer ou non, selon le mode « d'incorporation » des diverses composantes de la population qu'ils impliquent. Cette hypothèse s'inscrit dans le 
développement d'un cadre de réflexion sur les migrations, qui met paradoxalement l'accent sur la «non-migration».

48. - Ces conférences sont rapportées dans les numéros correspondants de la revue Al-'Amal, publiée chaque trimestre par le ministère du Travail.

49. -Durant les années quatre-vingt, le prince héritier Hassan, frère du roi Hussein s'en chargeait, par conviction personnelle plus que par désir de soutien politique du régime à l'événement.

50. -Les hauts niveaux remarqués en 1992-1993 correspondent au rapatriement des fonds des returnees vers les banques jordaniennes.

51. -Les données présentées ici concernent l'ensemble du Moyen-Orient, défini dans ces statistiques comme la région s'étendant du Maroc au Pakistan (inclus). Elles sont tirées de compilations des données du recensement de l'an 2000 aux États-Unis. La Jordanie n'apparaît nulle part dans ces compilations statistiques sur l'origine des migrants, en raison des faibles effectifs bruts de ses ressortissants.

52. - On se concentre sur ces deux pays, qui absorbent la plus grande part des migrants moyen-orientaux. Les migrations vers l'Europe sont peu importantes.

53. -L'éventualité d'un traitement différentiel des migrants originaires des pays arabes et / ou musulmans, d'une fermeture progressive des frontières à ces personnes, appelée par certains courants de l'opinion publique, ont été officiellement écartées par les autorités des deux pays. Des tentatives d'harmonisation des politiques d'immigration entre les Etats-Unis et le Canada n'ont pas abouti. Les mesures prises par le Canada restent limitées à une surveillance renforcée des frontières, à une lutte contre l'immigration clandestine et l'usage de faux documents. Les deux pays mènent une politique d'échange de renseignements sur les candidats à l'immigration (Adelman, 2002). Depuis le 28 juin 2002, la procédure de recrutement au Canada a subi une évolution, vers la mise en place de critères de sélection plus sévères, officiellement appliqués à l'ensemble des migrants. Le niveau d'éducation minimal requis est élevé, l'état matrimonial des candidats prend une importance, avec une préférence des autorités pour les personnes mariées. La raison invoquée reste, non les événements du 11 septembre et le problème du lien migrations-sécurité, mais celui de la forte augmentation du nombre des demandes (Singer, 2002).

54. -Pakistan non inclus.

55. -Un programme qui alloue chaque année 50000 visas par tirage au sort à des candidats de l'ensemble des pays du monde.

56. -La raison invoquée officiellement est celle de la protection des Palestiniens et de leurs biens face aux agressions israéliennes, de la prévention d'un transfert facile et « volontaire » des Palestiniens vers les pays arabes voisins : si les visiteurs temporaires sur la rive est du Jourdain se trouvaient empêchés par les autorités israéliennes de revenir vers la rive occidentale, la Jordanie se trouverait placée devant un fait accompli rappelant ceux intervenus au cours de l'histoire. Le débat reste en effet vif, à la suite des travaux des nouveaux historiens israéliens, sur les circonstances réelles de la « catastrophe » de 1948. La responsabilité de violences perpétrées à certaines occasions par les sionistes (massacre de Deir Yasin) dans l'exode massif des populations palestiniennes a été démontrée, mais certains nationalistes dans le monde arabe reprochent aux Palestiniens d'avoir cédé à l'intimidation, de n'avoir pas lutté suffisamment pour défendre leur terre. 
57. -La période de séjour sur la rive est est fixée en fonction du statut de résidence du demandeur (résidants en Jordanie ou en Cisjordanie). Ces derniers (détenteurs de cartes vertes) ne peuvent désormais rester en Jordanie que 48 heures au maximum. 58. -Par exemple : 11000 entrées au cours de la première année de la seconde Intifada pour 13000 retours (interview par Awadat, I. The Star, 8/8/2002). En contradiction avec ces chiffres, les écrits du nationaliste Fahd Al-Fanek dans le quotidien Al-Ra'i (journal contrôlé par l'État comme l'ensemble de la presse jordanienne) affirment qu'à la mi-2002, 40000 Palestiniens attendaient de passer la frontière. Le nombre d'entrées accepté par les autorités jordaniennes était alors de 1000 personnes par jour (Al-Ra'i, $15 / 7 / 2002$ ).

59. -Les données citées dans ce paragraphe sur les Irakiens en Jordanie sont tirées des travaux de cet auteur.

60. -Le visa attribué aux ressortissants irakiens n'est valable que six mois.

61. -Per capita output indicator, fondé sur l'indicateur du pouvoir d'achat élaboré par le PNUD (Purchasing Power Parity, (PPP)).

62. -Dans le sens donné à ce mot par le rapport du PNUD.

\section{RÉSUMÉS}

Cet article met en relief l'importance décisive des mouvements d'émigration internationale dans le processus de stabilisation politique en Jordanie jusqu'à nos jours. A deux périodes de crise, nées de flux massifs de population vers la Jordanie (1967 et 1990-91), répondent deux vagues d'émigration internationale, qui permettent d'éviter au régime une renégociation des liens de redistribution clientéliste des ressources et des modalités d'accès au capital. Les migrations sont donc un facteur constitutif du politique, en Jordanie comme dans d'autres pays arabes.

Or, si la demande d'émigration s'accentue, l'offre n'augmente pas, voire pourrait se réduire comme le laissent présager des mesures prises par des pays occidentaux depuis le 11 septembre 2001. Dans le même temps, le verrouillage politique s'accentue dans les pays arabes. On peut donc y craindre une transition démocratique troublée, à moins que ne s'y opère une réappropriation des ressources humaines auparavant évincées du processus de construction nationale et de développement par l'émigration.

International Migrations and Politics in Jordan. This article illustrates the role of international emigration movements in the process for political stability in Jordan. The two waves of Palestinian refugees to Jordan in 1967 and 1990-91 developed into domestic political crises, which were deflected by a new process of international re-emigration. Thus, the regime could avoid a reshuffling of the clientelist mode of access to capital. In Jordan, in as much as in other Arab countries, migration is a political factor.

However, migration demand increases while opportunities may decrease, as can be witnessed in measures taken by some western countries following September 11th, 2001. In the meantime, Arab states are tightening political control over their populations. One can thus fear a troubled democratic transition in the region, unless human resources, which were marginalized because of former emigration trends, can be reintegrated in the process of nation building and development. 
Migraciones internacionales y política en Jordania. Este artículo subraya la importancia decisiva de los movimientos de emigración internacional en los procesos de estabilización política de Jordania. A los dos períodos de crisis originados por flujos masivos de población palestina hacia Jordania (1967 y 1990-91), corresponden dos olas de emigración internacional. Dichas olas evitaron que el régimen se viera obligado a renegociar los lazos de redistribución clientelista de los recursos y de las modalidades de acceso al capital. Tanto en Jordania como en otros países árabes, las migraciones son un factor constitutivo de la política.

Ahora bien, mientras la demanda de emigración se acentúa, la oferta no aumenta sino que más bien se reduce, tal y como lo dejan presagiar las medidas tomadas por los países occidentales desde el 11 de septiembre. Si a esta situación se le suma el creciente enclavamiento político de los países árabes, podríamos imaginar una transición democrática inestable. La única alternativa pasaría por la reapropiación de los recursos humanos hasta ahora excluidos del proceso de construcción nacional y de desarrollo a través de la emigración.

\section{INDEX}

Mots-clés : politique d'immigration, besoin de main-d'œuvre, population déplacée

Index géographique : Jordanie

\section{AUTEUR}

\section{FRANÇOISE DE BEL-AIR}

Chercheur associée, Institut Français du Proche Orient, P.O.Box 830413 Zahran 11 183, Amman, Jordanie 